\title{
GRADED MESH APPROXIMATION IN WEIGHTED SOBOLEV SPACES AND ELLIPTIC EQUATIONS IN 2D
}

By

\section{James Adler and Victor Nistor}

\section{IMA Preprint Series \#2410}

(September 2012)

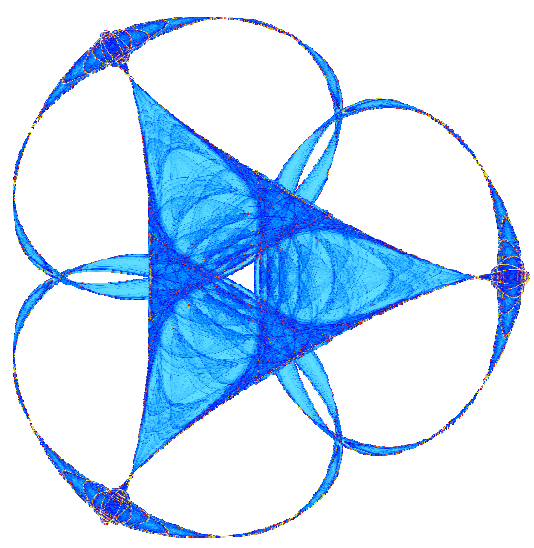

INSTITUTE FOR MATHEMATICS AND ITS APPLICATIONS

UNIVERSITY OF MINNESOTA 400 Lind Hall

207 Church Street S.E.

Minneapolis, Minnesota 55455-0436

Phone: 612-624-6066 Fax: 612-626-7370

URL: http://www.ima.umn.edu 


\title{
GRADED MESH APPROXIMATION IN WEIGHTED SOBOLEV SPACES AND ELLIPTIC EQUATIONS IN 2D
}

\author{
JAMES ADLER AND VICTOR NISTOR
}

\begin{abstract}
We study the approximation properties of some general finiteelement spaces constructed using improved graded meshes. In our results, either the approximating function or the function to be approximated (or both) are in a weighted Sobolev space. The finite-element spaces that we define are obtained from conformally invariant families of finite elements (no affine invariance is used), stressing the use of elements that lead to higher regularity finite-element spaces. We prove that for a suitable grading of the meshes, one obtains the usual optimal approximation results. We provide a construction of these spaces that does not lead to long, "skinny" triangles. Our results are then used to obtain $h^{m}$-quasi-optimal rates of convergence for the FEM approximation of solutions of strongly elliptic interface/boundary value problems.
\end{abstract}

\section{INTRODUCTION}

Consider the typical problem of approximating the solutions of a diffusion problem with zero Dirichlet boundary conditions,

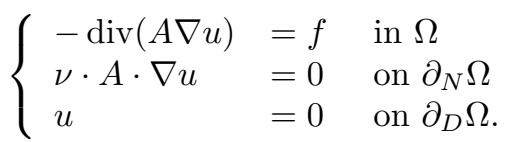

Here, $\nu$ is the outward normal vector to the boundary, $\Omega$ is a polygonal domain in two space dimensions (2D) or three space dimensions (3D), and $f \in H^{m-1}(\Omega)$. We allow piecewise smooth coefficients, so we consider also transmission problems. This problem arises in many practical applications. Typically one is interested in approximating the solution, $u$, with some simpler functions or at least in approximately computing some quantities of interest associated to $u$. One of the most commonly used methods to approximate $u$ is the Finite-Element Method (FEM). See for instance $[8,14,17,21,37,38]$ for introductions to this method. The results here extend almost without change to systems such as the elasticity system and to non-homogeneous boundary conditions.

The standard applications of FEM to approximate the solution, $u$, of Equation (1) requires it to possess good regularity properties. However, it is known [7, 26, $27,30]$ that the solution, $u$, of this equation on non-smooth domains is typically not in $H^{m+1}(\Omega)$, but rather has the limited regularity, $u \in H^{s-\epsilon}(\Omega)$, where $s=s_{\Omega}$ is a constant associated to $\Omega$ and where $\epsilon>0$ is arbitrary. This is not an artifact of the method, but it does lead to decreased rates of convergence in the FEM approximations. Due to its practical importance, many works have been devoted

\footnotetext{
V.N. was partially supported by NSF Grants OCI-0749202 and DMS-1016556.
} 
to correcting this deficiency, see for example $[1,4,9,10,11,12,14,25,23,13,28$, $32,35]$.

A common result of the above research is that the solution, $u$, of equation (1) has better regularity properties in a weighted Sobolev space [30, 31, 35]. More precisely, let $r_{\Omega}(x)$ denote the distance from $x$ to the set of singular points of $\Omega$ [35] and define the weighted Sobolev space of order $m$ and weight $a$ by

$$
\mathcal{K}_{a}^{m}(\Omega)=\left\{v: \Omega \rightarrow \mathbb{C}, r_{\Omega}^{|\alpha|-a} \partial^{\alpha} v \in L^{2}(\Omega),|\alpha| \leq m\right\} .
$$

Then, $u \in \mathcal{K}_{a+1}^{m+1}(\Omega)$, for $a>0$ small enough (more precisely, $a<\pi / \alpha_{\max }$, where $\alpha_{\max } \in(0,2 \pi]$ is the maximum angle of $\left.\Omega\right)$. In two dimensions, this is enough to recover quasi-optimal rates of convergence for the finite-element approximations of $u[1,9,12,35]$. The proof of this result depends essentially on an approximation property of the solution, $u$, in weighted Sobolev spaces using graded meshes. More precisely, it was shown (see for example [3, 9, 12, 35]) that there exists a sequence of nested meshes, $\mathcal{T}_{n}$, with the following property: Let $S_{n}$ be the sequence of finite-element spaces of continuous functions on $\Omega$ that restrict to each triangle of $\mathcal{T}_{n}$ a polynomial of order $m$. Then, $\operatorname{dim}\left(S_{n}\right) \rightarrow \infty$ and there exists a sequence of interpolation operators $I_{n}$ and a constant $C>0$ with the property $\left\|u-I_{n}(u)\right\|_{H^{1}(\Omega)} \leq C \operatorname{dim}\left(S_{n}\right)^{-m / 2}\|u\|_{\mathcal{K}_{a+1}^{m+1}(\Omega)}$, for $m \geq 1$. Since there exists a constant $C$ such that $\|u\|_{\mathcal{K}_{a+1}^{m+1}(\Omega)} \leq C\|f\|_{H^{m-1}(\Omega)}$, the above approximation becomes

$$
\left\|u-I_{n}(u)\right\|_{H^{1}(\Omega)} \leq C_{a} \operatorname{dim}\left(S_{n}\right)^{-m / 2}\|f\|_{H^{m-1}(\Omega)},
$$

for some constant $C_{a}>0$. This is the same result that one would obtain in the classical case of quasi-uniform meshes, if the solution $u$ were in $H^{m+1}(\Omega)$.

In this paper, we extend the approximation result of Equation (3) above and offer different variants of the construction of the sequence of graded meshes $\mathcal{T}_{n}$. For instance, we offer a construction that yields a minimum angle condition independent of $m$. This is relevant for the $h p$-version of the FEM as long skinny triangles are avoided. We also include interfaces, in the sense that we partition the domain, $\Omega$, into several polygonal subdomains, $\Omega_{j}$, and we only assume $u \in \mathcal{K}_{a+1}^{m+1}\left(\Omega_{j}\right)$ for all $j$, but $u \in \mathcal{K}_{a+1}^{1}(\Omega)$ for the entire domain.

Another new feature of this paper is that in it we consider finite-element spaces with higher regularity, forcing us to consider finite-element spaces generated by conformally invariant families (this definition is slightly more general than the one in Section 2.3 of [21], for instance). Therefore, the constructions are not restricted to Lagrange finite elements. This may be useful for higher order problems. See $[18,16,29]$ for example. In addition, we provide $L^{2}$-error estimates.

To formulate more precisely the general form of the problem, assume that the straight polygonal domain, $\Omega$, is decomposed as $\bar{\Omega}=\cup_{k=1}^{K} \bar{\Omega}_{k}$, where $\Omega_{k}$ are disjoint straight polygonal domains. The set $\Gamma:=\partial \Omega \backslash \cup_{k=1}^{K} \partial \Omega_{k}$, that is, the part of the boundary of some $\Omega_{k}$ that is not contained in the boundary of $\Omega$, is called the interface, as usual. Then, assume that the coefficients $A=\left[a_{i j}\right]$ have only jump discontinuities across the interface $\Gamma$. That is, the restriction to any of the domains, $\Omega_{k}$, of any of the coefficients, $a_{i j}$, extends to a smooth function on $\bar{\Omega}_{k}$. Also, Equation (1) is formulated in a weak sense, which implies the usual matching and jump conditions at the interface. (See Section 3 for details.) We also assume that $-\operatorname{div}(A \nabla)$ is uniformly strongly elliptic, in the usual sense, see Equation (31). 
The paper is organized as follows. In Section 1, we describe the family of finite elements that we consider as well as stating the outline of the problem. In Section 2, we state and prove the approximation results. In addition, we describe the graded meshes and how they produce optimal results. Section 3 gives examples on how the above results can be applied to finite-element discretizations for certain problems. We also explain how to obtain classical "textbook" $h^{m}$-error estimates. Finally, we make some concluding remarks toward the end of the paper.

Acknowledgements. We thank Douglas Arnold, Hengguang Li, Tom Manteuffel, and Chad Westphal for useful discussions as well as Tufts University for seed funding of collaborative visits.

\section{Conformal Families of Finite Elements}

We consider a bounded polygonal domain, $\Omega \subset \mathbb{R}^{2}$, with straight edges. We also assume that we are given a disjoint decomposition of the boundary into "Dirichlet" and "Neumann" parts, $\partial \Omega=\partial_{D} \Omega \cup \partial_{N} \Omega$, with $\partial_{D} \Omega$ a closed subset, and both $\partial_{D} \Omega$ and $\partial_{N} \Omega$ sets with finitely many components.

This section explains the type of finite elements considered in this paper. The reader may skip this section at the first reading and go to the next section, assuming for instance that order $m$ Lagrange elements are used, in which case this construction is simpler.

1.1. A Typical Finite Element. Consider (essentially) the framework of Ciarlet $[22,21]$. The main difference in our approach is that we emphasize more the interpolant rather than the degrees of freedom. Also, our families will not be, in general, affine equivalent, so we do not consider reference finite elements.

Consider an arbitrary triangle, $T$, fixed throughout this subsection. Denote by $\mathcal{P}_{j}$, the space of polynomials $P: \mathbb{R}^{2} \rightarrow \mathbb{R}$ of degree (at most) $j$, and fix a dimension$N$ space $\mathcal{P}_{T}$,

$$
\mathcal{P}_{m} \subset \mathcal{P}_{T} \subset \mathcal{P}_{m^{\prime}} .
$$

In addition to the integer parameters $m$ and $m^{\prime}$ introduced above in Equation (4), we also fix two integers $r \leq s$. The integer $s$ determines the smoothness of the functions that can be interpolated (by the higher order of the derivatives appearing among the degrees of freedom) and is called the degree of the interpolant. The integer $r$ is defined such that the resulting finite-element (FE) space has smoothness $H^{r+1}$. The integer $m$ is called the polynomial degree of approximation and the integer $m^{\prime}$ is called the maximum polynomial degree of the FE spaces.

Then, consider linear functionals, $\ell_{i}$, on $\mathcal{C}^{s}\left(\mathbb{R}^{2}\right), 1 \leq i \leq N=\operatorname{dim}\left(\mathcal{P}_{T}\right)$, called degrees of freedom, whose restrictions to $\mathcal{P}_{T}$ form a basis of $\mathcal{P}_{T}^{*}$, where $\mathcal{P}_{T}^{*}$ denotes the space of linear functionals on $\mathcal{P}_{T}$. Assume that the degrees of freedom are of one of the following three types, with $p$ and $q$ denoting polynomials:

$$
\begin{gathered}
\ell_{i}: \mathcal{P}_{T} \rightarrow \mathbb{R}, \quad \ell_{i}(p)=\partial_{x}^{j} \partial_{y}^{k} p(z), \quad \text { where } z \in T \text { and } j+k \leq s, \\
\ell_{i}(p)=\int_{\sigma} q(x, y) \partial_{\nu}^{k} p(x, y) d \sigma, \quad \text { where } \sigma=\text { an edge of } T \text { and } k \leq s, \text { or } \\
\ell_{i}(p)=\int_{T} q(x, y) p(x, y) d x d y .
\end{gathered}
$$

If $\ell_{i}$ is of the first type (type I), then we say that its support is $z$. If $\ell_{i}$ is of the second type (type II), then we say that its support is $\sigma$. Finally, if $\ell_{i}$ is of the third 
type (type III), we say that its support is $T$. Thus, the support of $\ell_{i}$ is the support of the corresponding distribution. If $\ell_{i}$ is of type II, then $\nu$ is a unit normal vector to $\sigma$ and $d \sigma$ is the arc length measure on $\sigma$. The motivation for considering degrees of freedom that are not type I is provided, for instance, by $[5,6]$.

Denote by $\Sigma_{T}:=\left\{\ell_{i}\right\}$ the set of the given linear functionals, which are assumed to be a linearly-independent set. Since $\Sigma_{T}$ is a basis of $\mathcal{P}_{T}^{*}$, there exists a dual basis $\left\{p_{i}\right\} \subset \mathcal{P}_{T}$ such that

$$
\ell_{i}\left(p_{j}\right)=\delta_{i j},
$$

and, then, we define the interpolation operator as usual,

$$
I_{T}=I_{T, \mathcal{P}_{T}, \Sigma}: \mathcal{C}^{s}(\bar{T}) \rightarrow \mathcal{P}_{T}, \quad I_{T}(f)=\sum_{i=1}^{N} \ell_{i}(f) p_{i} .
$$

(Recall that $\delta_{i j}$ denotes the Kronecker symbol: $\delta_{i i}=1$ and $\delta_{i j}=0$ if $i \neq j$.) The fact that $\left\{p_{i}\right\}$ is the dual basis to $\left\{\ell_{i}\right\}$ ensures that $I_{T}^{2}=I_{T}$, and, hence, $I_{T}$ is a projection onto $\mathcal{P}_{T}$, that is $I_{T}(p)=p$, for all $p \in \mathcal{P}_{T}$. Then, $\Xi_{T}:=\left(T, \mathcal{P}_{T}, I_{T}\right)$ is a finite element on $T$. The support of $\Xi_{T}$ is the set consisting of the supports of the degrees of freedom $\ell_{i} \in \Sigma_{T}$. Note, however, that the set $\Sigma_{T}$ is not determined by the triple $\Xi_{T}:=\left(T, \mathcal{P}_{T}, I_{T}\right)$, in the sense that the same finite element can be obtained from a different choice of degrees of freedom. It is in this sense that we choose to make $I_{T}$ a more important ingredient of the definition than the set $\Sigma_{T}$. The reason for this choice is the better invariance properties of the interpolation operator $I_{T}$, see below. This is the main difference to [21]. Finally, note that the support of $\Xi_{T}$ is independent on the choice of the degrees of freedom.

If $T^{\prime}$ is any other triangle and $L$ is an invertible affine map (that is a linear map plus a translation) such that $L(T)=T^{\prime}$, then $L$ can transport $\mathcal{P}_{T}, I_{T}$, and the support of $\Xi_{T}=\left(T, \mathcal{P}_{T}, I_{T}\right)$ to $T^{\prime}$ as usual. Explicitly, let

$$
\mathcal{P}_{T^{\prime}}:=\left\{p \circ L^{-1}, p \in \mathcal{P}_{T}\right\} \text { and } I_{T}(f)=I_{T}(f \circ L) \circ L^{-1} .
$$

Then, we denote by

$$
L\left(\Xi_{T}\right):=\Xi_{T^{\prime}}=\left(T^{\prime}, \mathcal{P}_{T^{\prime}}, I_{T^{\prime}}\right),
$$

the corresponding finite element on $T^{\prime}$.

1.2. Assumptions on the Families of Finite Elements. Assume that to each triangle, $T$, in the plane there is associated a finite element $\Xi_{T}=\left(T, \mathcal{P}_{T}, I_{T}\right)$ and that this family depends continuously on $T$ (in an obvious sense, see Assumption 1 ). Notice that the continuity condition implies the continuity of the supports of the elements, $\Xi_{T}$. In particular, if one of the finite elements has an edge in its support, then all the edges of all triangles are in the supports of the corresponding $\Xi_{T}$. The following three assumptions are made for this family, $\mathcal{F}=\left\{\Xi_{T}\right\}$, of finite elements. First, in order to obtain interpolation estimates in the usual way, we assume that the family is continuous and conformally invariant.

Assumption 1: Continuity and conformal invariance. The family $\mathcal{F}=\left\{\Xi_{T}\right\}$, $\Xi_{T}=\left(T, \mathcal{P}_{T}, I_{T}\right)$, is continuous and conformally invariant in the following sense. First, the spaces, $\mathcal{P}_{T} \subset \mathcal{P}_{m^{\prime}}$, and the interpolants, $I_{T}: \mathcal{C}^{s}\left(\mathbb{R}^{2}\right) \rightarrow \mathbb{R}$, depend continuously on $T$ and, second, if $L$ is a conformal linear map (the composition of dilations and isometries) and $L(T)=T^{\prime}$, then $L\left(\Xi_{T}\right)=\Xi_{T^{\prime}}$.

Next, we want the resulting finite-element spaces to have smoothness $\mathcal{C}^{r}$. 
Assumption 2: Matching of derivatives. Let $\sigma$ be an edge and choose any triangle $T$ with $\sigma$ as an edge. Also, denote by $\Sigma_{T, \sigma}$ the set of degrees of freedom $\ell_{i} \in \Sigma_{T}$ with support contained in $\sigma$. We assume that if $\ell_{i}(p)=0$ for all $\ell_{i} \in \Sigma_{T, \sigma}$, then $\partial_{\nu}^{k} p=0$ on $\sigma$ for all $k \leq r$ (here, as before, $\nu$ is the normal derivative to $\sigma$ ).

The third assumption has to do with constructing degrees of freedom of the associated finite-element space. Let $T$ be a triangle with associated finite element $\Xi_{T}$. Fix a set of degrees of freedom, $\Sigma_{T}$, defining $\Xi_{T}$. We shall regard $\Sigma_{T}$ as a subset of the space, $\mathcal{C}^{s}\left(\mathbb{R}^{2}\right)^{\prime}$, of continuous linear maps, $\mathcal{C}^{s}\left(\mathbb{R}^{2}\right) \rightarrow \mathbb{R}$. In order to formulate the assumption on the meshes, we need to introduce the following notation:

(1) Let $z$ be a vertex of $T$, then denote $V_{z}$ as the linear span of the degrees of freedom supported on $z$.

(2) Let $\sigma$ be an edge of $T$, then denote $V_{\sigma}$ as the linear span of the set of degrees of freedom of $\Sigma_{T}$ supported on $\sigma$.

(3) Let $\sigma$ be an edge of $T$ and $z \in \sigma$ be a point that is not a vertex of $\sigma$, then denote $V_{\sigma, z}$ as the linear span of the set of degrees of freedom of $\Sigma_{T}$ supported on $z$.

(4) Finally, denote $V_{T}$ as the set of degrees of freedom of $\Sigma_{T}$ supported on $T$. Then, the third assumption on the family $\mathcal{F}$ of finite elements is as follows.

Assumption 3: Independence of degrees of freedom. The sets $V_{z}, V_{\sigma}$, and $V_{\sigma, z}$, introduced above, do not depend on $T$ (they depend only on the indicated subscripts).

1.3. Examples of Families of Finite Elements. We now construct examples of families, $\mathcal{F}=\left\{\Xi_{T}\right\}, \Xi_{T}=\left(T, \mathcal{P}_{T}, I_{T}\right)$, of finite elements satisfying the three assumptions of the previous subsection, where $T$ ranges through the set of all triangles.

The most important example is also the simplest: Lagrange triangles of type $(m)$ [21]. Recall that in this case, $m=m^{\prime}$ and $r=s=0$, so $\mathcal{P}_{T}=\mathcal{P}_{m}$ for any triangle $T$. The degrees of freedom are given by the evaluations at the points $z \in T$ that, in barycentric coordinates on $T$, are of the form $\left[\lambda_{0} / m, \lambda_{1} / m, \lambda_{2} / m\right]$, where $\lambda_{1}, \lambda_{2}$, and $\lambda_{3}$ are integers. See [21] for a proof that all the conditions are satisfied for this finite element. In fact, these families are even affine invariant (not just conformally invariant).

Higher regularity finite-element spaces are needed for fourth order problems and in certain formulations of second-order elliptic problems using the least-squares finite-element method. Two examples that yield $\mathcal{C}^{1}$-finite elements are the Argyris and the Bell triangle [21]. The Argyris triangle provides an example with $m=$ $m^{\prime}=5, s=1$, and $r=2$. The degrees of freedom are all the partial derivatives of order $\leq r=2$ at the vertices and the normal derivative at the midpoints of the edges (so $N=21)$. The Bell triangle is similar, but $m=4\left(m^{\prime}, s\right.$, and $r$ are the same). Both the Argyris and Bell triangles require $\mathcal{C}^{2}$-regularity of the function to be approximated (in the domain of the interpolant), while yielding only $\mathcal{C}^{1}$-regularity for the resulting finite-element space.

1.4. Finite-Element Space and Interpolation. With the notation and the assumptions of Subsection 1.2, let $\mathcal{F}$ denote the given family of finite elements, $\Xi_{T}$. We now extend the constructions of that subsection to a mesh, $\mathcal{T}=\left\{T_{j}\right\}$, on $\Omega$ yielding a finite-element space, $S(\mathcal{T}, \mathcal{F})=S(\mathcal{T})$, and an interpolation operator, 
$I_{\mathcal{T}, \mathcal{F}}=I_{\mathcal{T}}$, as follows. Recall, that a mesh $\mathcal{T}$ on $\Omega$ is a set of disjoint (open) triangles, $T_{j} \subset \Omega$, satisfying $\cup \bar{T}_{j}=\bar{\Omega}$. We also assume that $\partial_{D} \Omega$, the Dirichlet part of the boundary, is a union of edges of triangles, $T \in \mathcal{T}$. Hence, the Neumann part of the boundary, $\partial_{N} \Omega$, has the same property. Eventually (beginning with Section 2 ), we require the triangles of $\mathcal{T}$ to be aligned to the interface. For this discussion, though, this is not necessary. We also assume that the meshes are conforming, meaning, as usual, that if the closures $\overline{T_{i}}$ and $\overline{T_{j}}$ of two triangles $T_{i}$ and $T_{j}$ of this mesh intersect, then their intersection $\overline{T_{i}} \cap \overline{T_{j}}$ is either a vertex or an edge of these triangles.

Consider for each triangle, $T$, the space $\mathcal{P}_{T}$ and the support of the finite element $\Xi_{T}$. The union of the supports of all the finite elements, $\Xi_{T}$, is the support of $\mathcal{T}$. Recall the notation introduced in Subsection 1.2 and consider the following. For each vertex $z$ of $\mathcal{T}$, consider a basis $\Sigma_{z}$ of the set $V_{z}$. For each edge $\sigma$ of $\mathcal{T}$, consider a basis $\Sigma_{\sigma}$ of $V_{\sigma}$. Finally, for each edge $\sigma$ of $\mathcal{T}$ and $z \in \sigma$ that is the support of some degree of freedom, consider a basis $\Sigma_{\sigma, z}$ of $V_{\sigma, z}$. Notice that the sets $\Sigma_{z}, \Sigma_{\sigma}$, and $\Sigma_{\sigma, z}$ are defined using a triangle $T$ of the mesh. However, by Assumption 3 of Subsection 1.2, these sets do not depend on the choice of $T$. Then, the set $\Sigma_{\mathcal{T}, \mathcal{F}}$ of degrees of freedom associated to $\mathcal{T}$ and $\mathcal{F}$ is defined as the union of the sets $\Sigma_{z}$, $\Sigma_{\sigma}, \Sigma_{\sigma, z}$ considered above, and of the basis of all the spaces $V_{T}$, where $T$ ranges through all the triangles of $\mathcal{T}$.

We now define the finite-element space, $S(\mathcal{T})=S(\mathcal{T}, \mathcal{F})$, associated to $\mathcal{F}:=$ $\left\{\Xi_{T}=\left(T, \mathcal{P}_{T}, \Sigma_{T}\right)\right\}$ and $\mathcal{T}$ as follows. Consider the set of families, $\left(f_{T}\right), f_{T} \in \mathcal{P}_{T}$, for all $T \in \mathcal{T}$. Such a family, $\left(f_{T}\right)$, is called matching if $\ell_{i}\left(f_{T}\right)=\ell_{i}\left(f_{T^{\prime}}\right)$ and if $T$ and $T^{\prime}$ are two adjacent triangles of $\mathcal{T}$, such that $\ell_{i} \in \Sigma_{\mathcal{T}, \mathcal{F}}$ is a degree of freedom that is common to both $T$ and $T^{\prime}$. Then,

$$
S(\mathcal{T}, \mathcal{F}):=\left\{\left(f_{T}\right), f_{T} \in \mathcal{P}_{T} \text { is a matching family and } f_{T}=0 \text { on } \partial_{D} \Omega\right\} .
$$

Let $u \in \mathcal{C}^{s}(\Omega)$. Then, for each triangle $T$ in the mesh $\mathcal{T}$, the interpolant $u_{T}:=$ $I_{T}(u) \in \mathcal{P}_{T}$ is defined, where $I_{T}$ is associated to $T$ and the family $\mathcal{F}:=\left\{\Xi_{T}\right\}$, $\Xi_{T}=\left(T, \mathcal{P}_{T}, I_{T}\right)$, as before. The assumptions from Subsection 1.2 on the finite element shows that the collection $\left(u_{T}\right)$ is a matching family, so it is in $S(\mathcal{F})$. We then define

$$
I_{\mathcal{T}, \mathcal{F}}(u)=\left(I_{T}(u)\right) \in S(\mathcal{T}, \mathcal{F}) .
$$

\section{Discretization Error Estimates and $\kappa$-Refinements}

The purpose of this section is to describe a sequence of (graded) triangular meshes, $\mathcal{T}_{n}$, in the domain, $\Omega$, that provide quasi-optimal approximations of functions in suitable weighted Sobolev spaces. In particular, we extend the approximation results of $[12,35]$ from Lagrange elements to the more general elements described above. We also consider approximations in other norms. Let $m \leq m^{\prime}$ and $r \leq s$ be as in the previous section. That is, the integer $m$ is the polynomial degree of approximation, the integer $m^{\prime}$ is the maximum polynomial degree of the FE spaces, the integer $s$ is the degree of the interpolant, and the integer $r+1$ is the smoothness of the FE space. Also, we consider a continuous family, $\mathcal{F}$, of conformally invariant finite elements, as described above in Subsection 1.2.

Next, consider the interfaces. Recall that the domain, $\Omega$, is a polygonal domain with straight edges (called a straight polygon). For simplicity, we do not allow for cracks or vertices that touch the boundary. The case of cracks would be very similar 
to that of the interface, but would allow functions with jump discontinuities along the crack. We leave for the reader to make the necessary changes to deal with cracks. On the other hand, when considering mixed boundary conditions, it is well known that singularities appear at the points where the boundary conditions change (from Dirichlet to Neumann). These singularities are very similar in structure to the singularities that appear at geometric vertices. Thus, we define the set $\mathcal{V}$ of singular points of $\Omega$ as the set where singularities of the solutions of elliptic partial differential equations may arise. These consist, in this case, of all the geometric vertices of $\Omega$, all points where the type of boundary conditions change, all points where the interface touches the boundary, and all the non-smooth points of the interface (more singular points would have to be included if cracks were allowed, such as the tips and ends of the cracks and the non-smooth points of the cracks).

Assume that $\bar{\Omega}=\cup_{j=1}^{K} \overline{\Omega_{j}}$, with $\Omega_{j}$ also straight polygons. Assume the domains, $\Omega_{j}$, are open and disjoint. We are looking to approximate a function $u \in \mathcal{K}_{a+b+1}^{1}(\Omega)$, such that the restriction of $u$ to any of the subdomains, $\Omega_{j}$, satisfies $u \in \mathcal{K}_{a+b+1}^{m+1}\left(\Omega_{j}\right)$ for all $j$. Here, $a>0$ is a constant that depends on the Problem (1) (for instance on the domain $\Omega$ ), whereas $b \in \mathbb{R}$ is a parameter that is considered in view of further applications, including the least squares finite-element method [32]. It is, therefore, convenient to assume that the initial refinement of $\Omega$ is such that the interface is resolved exactly by the meshes, that is, the interface is a union of the edges of the meshes that are considered. It turns out that it is enough to assume that the initial mesh, $\mathcal{T}_{0}$, resolves the interface exactly.

For the remaining part of this paper, we assume that the boundary of $\Omega$ and the interface $\Gamma$ are piecewise linear. For simplicity of the theoretical analysis, we again assume that there are no cracks. We note, however, that the mesh refinement in the case of cracks is completely similar, as long as one resolves the crack exactly and allows for discontinuous approximation functions along it.

2.1. Approximation Away from the Singular Points. We start by discussing the simpler approximation of the solution, $u$, away from the singular points. Recall that all estimates in the spaces $\mathcal{K}_{a}^{m}(\Omega)$ localize to subsets of $\Omega$.

Let $\mathbb{P}$ be any polygonal domain. In the applications considered here, $\mathbb{P}$ is a subset of $\Omega$. Let $\mathcal{T}$ be a mesh of $\mathbb{P}$ and let $S(\mathcal{T}, \mathcal{F})$ be the associated finite-element space as described in the previous section, Section 1. By a mesh or a triangulation of $\mathbb{P}$ we shall mean the same thing, since we only consider conforming meshes.

We denote by $u_{I}=I_{\mathcal{T}, \mathcal{F}}(u) \in S(\mathcal{T}, \mathcal{F})$ the interpolant of $u$. The interpolant $I_{\mathcal{T}}(u)=I_{\mathcal{T}, \mathcal{F}}(u)$ has the following approximation property that generalizes classical results from $[8,17,21,37]$. Many of the results below hold in greater dimensions, so we introduce $d$ to be the dimension of the domain, assuming though that $d=2$ in this paper.

Theorem 2.1. Let $\alpha>0$ and $0 \leq q \leq p \leq m+1$ be fixed integers, with $m$ as in Equation (4), $p>s+1=s+d / 2$ and $q \leq r+1$. Then, there exists a constant $C(\alpha, m)>0$ with the following property. Let $\mathcal{T}$ be a triangulation of $\mathbb{P}$ and assume that all triangles $T$ in $\mathcal{T}$ have angles $\geq \alpha$ and sides of length $\leq h$. Then, the interpolant $u_{I}:=I_{\mathcal{T}, \mathcal{F}}(u) \in S(\mathcal{T}, \mathcal{F})$ satisfies

$$
\left|u-u_{I}\right|_{H^{q}(\mathbb{P})} \leq C(\alpha, m) h^{p-q}|u|_{H^{p}(\mathbb{P})},
$$

for all $u \in H^{p}(\mathbb{P})$. 
This result is well known for affine invariant families [17, 21]. The main point of this proof is to extend it to conformally invariant families of finite elements.

Proof. Note that for $d \geq 2$ the relation $r \leq s$ gives $q<p$.

Consider a triangle $O A B$ as shown in the left side of Figure 2.1 and denote by $\hat{O}, \hat{A}$, and $\hat{B}$ the measures of its angles. Denote by $\mathcal{S}$ the set of triangles $O A B$ with the following properties:

(1) $O=(0,0)$ is the origin,

(2) $A=(x, 0)$ is on the positive $x$-axis $(x>0)$,

(3) the angles of the triangle satisfy $\hat{O} \leq \hat{A} \leq \hat{B}$ ( and

(4) $B$ is in the upper half-plane.

Then, any triangle $T$ in the plane is congruent to a unique triangle $T_{1}$ in $\mathcal{S}$. Denote the set of triangles $O A B$ in $\mathcal{S}$ for which $|O A|=x$ by $\mathcal{S}_{x}$. Since the set of conformal mappings contains the set of dilations, we have that every triangle $T$ is conformally equivalent to a triangle $T_{1} \in \mathcal{S}_{1}$. The vertex $B$ then completely determines the triangle $O A B$ in $\mathcal{S}_{1}$ (since $O$ and $A$ are fixed). Therefore, the set $\mathcal{S}_{1}$ identifies with the set

$$
\mathcal{B}:=\left\{B=(x, y) \in \mathbb{R}^{d}, y>0, x^{2}+y^{2} \leq 1, \text { and }(x-1)^{2}+y^{2} \leq 1\right\} .
$$

The set of triangles in $\mathcal{S}_{1}$ that have all angles $\geq \alpha$ hence form a compact set $K_{\alpha} \subset \mathcal{S}_{1}$. See right side of Figure 2.1.

Since $H^{p}\left(\mathbb{R}^{2}\right) \subset \mathcal{C}^{s}\left(\mathbb{R}^{2}\right)$, for each triangle $T \in \mathcal{S}$, the interpolation map gives rise to a continuous, linear map $I_{T}: H^{p}\left(\mathbb{R}^{2}\right) \rightarrow \mathcal{P}_{m^{\prime}}$. The function

$$
\mathcal{S} \ni T \rightarrow I_{T} \in \mathcal{L}\left(H^{p}, \mathcal{P}_{m^{\prime}}\right),
$$

is then a continuous function, by the assumption that the family $\mathcal{F}$ of finite elements is continuous. Since the range of each of the interpolants $I_{T}$ contains the space $\mathcal{P}_{m}$ of polynomials of degree $\leq m$, we have that

$$
\left|u-I_{T}(u)\right|_{H^{q}(T)} \leq C_{T}|u|_{H^{p}(T)},
$$

by the Bramble-Hilbert Lemma $[17,21]$. The continuity of the family of interpolants $I_{T}$ and the compactness of the set $K_{\alpha}$ implies that the constant $C_{T}$ in Equation (12) can be chosen to be independent of $T \in K_{\alpha} \subset \mathcal{S}_{1}$ and, hence, to depend only on $\alpha$ and $q<p \leq m$.

Next, if $T$ is any triangle in $\mathcal{S}_{x}, x \leq h$, with all angles $\geq \alpha$, the dilation invariance of the interpolation $I_{T}$ and of the semi norms $|u|_{H^{q}(T)}$ and $|u|_{H^{p}(T)}$ (up to a factor) gives

$$
\left|u-I_{T}(u)\right|_{H^{q}(T)} \leq C(\alpha, m) x^{p-q}|u|_{H^{p}(T)} \leq C(\alpha, m) h^{p-q}|u|_{H^{p}(T)},
$$

since $x=|O A|$ is the diameter of the triangle $T$. The invariance of all the terms of Equation (13) under isometries then gives the same result for all triangles with angles $\leq \alpha$ and sides $\leq h$. Adding together the squares of all the estimates (13) for all the triangles in $\mathcal{T}$ then gives the desired result.

We also obtain the following usual estimate.

Corollary 2.2. Using the assumptions and notation of Theorem 2.1, there exists a constant $C=C(\alpha, m)$ such that

$$
\left\|u-u_{I}\right\|_{H^{q}(\mathbb{P})} \leq C(\alpha, m) h^{p-q}\|u\|_{H^{p}(\mathbb{P})} .
$$



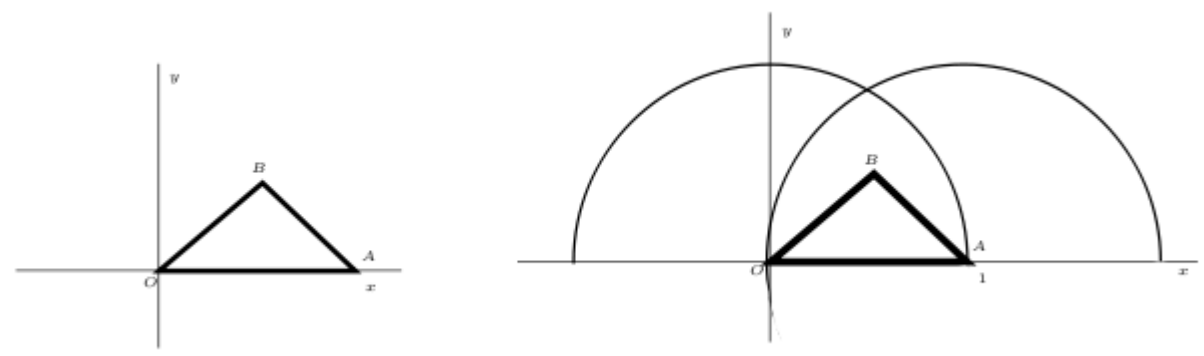

Figure 1. Left Side: Sample triangle in $\mathcal{T}$. Right Side: Depiction of the set, $\mathcal{S}_{1}$.

for all $u \in H^{p}(\Omega)$.

Proof. The proof follows by adding all the squares of the estimates of Theorem 2.1 for $(q, p)$ replaced by $(q-j, p-j), j=0, \ldots, q$.

The following estimate for the interpolation error on a proper subdomain of $\Omega$ (i. e., not at a positive distance from the corners) then follows from the equivalence of the $H^{m}(\Omega)$-norm and the $\mathcal{K}_{a}^{m}(\Omega)$-norm on proper subsets of $\Omega$. Recall that $r_{\Omega}(x)$ denotes the distance from $x$ to the singular points of $\Omega$, as in the introduction. If $G$ is an open subset of $\Omega$, define

$$
\mathcal{K}_{a}^{m}\left(G ; r_{\Omega}\right):=\left\{f: \Omega \rightarrow \mathbb{C}, r_{\Omega}^{|\alpha|-a} \partial^{\alpha} f \in L^{2}(G), \text { for all }|\alpha| \leq m\right\},
$$

and let $\|u\|_{\mathcal{K}_{a}^{m}(G)}$ denote the corresponding norm:

$$
\|u\|_{\mathcal{K}_{a}^{m}(G)}^{2}=\sum_{|\alpha| \leq m}\left\|r_{\Omega}^{|\alpha|-a} \partial^{\alpha} f\right\|_{L^{2}(G)}^{2}
$$

Note that this definition is similar to that of the usual weighted Sobolev spaces $\mathcal{K}_{a}^{m}(\Omega)$ introduced in Equation (2). In particular, $\mathcal{K}_{a}^{m}(\Omega)=\mathcal{K}_{a}^{m}\left(\Omega, r_{\Omega}\right)$. Also, we write $\mathcal{K}_{a}^{m}\left(G ; r_{\Omega}\right)=\mathcal{K}_{a}^{m}(G)$ when convenient.

Proposition 2.3. Fix $\alpha>0, \xi>0$, and integers $0 \leq q \leq p \leq m+1$ such that $p>s+d / 2$ and $q \leq r+1$. Let $G \subset \Omega$ be an open subset such that $r_{\Omega}>\xi$ on $G$ and let $\mathcal{T}=\left(T_{j}\right)$ be a triangulation of $\Omega$ with angles $\geq \alpha$ and sides $\leq h$. Then, for any given weights $a, b \in \mathbb{R}$, there exists a constant $C=C(\alpha, m, \xi, a, b)>0$ such that

$$
\left\|u-u_{I}\right\|_{\mathcal{K}_{b}^{q}(G)} \leq C h^{p-q}\|u\|_{\mathcal{K}_{a}^{p}(G)}, \quad \forall u \in \mathcal{K}_{a}^{p}(\Omega) .
$$

Proof. This follows from Theorem 2.1 and the equivalence of the $H^{t}$ and $\mathcal{K}_{a}^{t}$ norms on $\mathcal{K}_{a}^{t}\left(G ; r_{\Omega}\right)$, for any $t$ and $a$.

Similarly, we introduce a version of the $L^{\infty}$ weighted Sobolev spaces by

$$
\mathcal{W}^{m, \infty}\left(G ; r_{\Omega}\right):=\left\{v: G \rightarrow \mathbb{R}, r_{\Omega}^{|\alpha|} \partial^{\alpha} v \in L^{\infty}(G), \text { for all }|\alpha| \leq m\right\},
$$

with norm

$$
\|v\|_{\mathcal{W}^{m, \infty}(G)}=\sum_{|\alpha| \leq m}\left\|r_{\Omega}^{|\alpha|} \partial^{\alpha} v\right\|_{L^{\infty}(G)} .
$$

We sometimes write $\mathcal{W}^{m, \infty}(G)=\mathcal{W}^{m, \infty}\left(G ; r_{\Omega}\right)$. Also, when the domain is clear, we omit it from the notation of the norm. 
Then, we have the following lemma, whose proof is a direct calculation.

Lemma 2.4. There exist absolute constants $C_{m}, m \geq 0$, such that $\|v u\|_{\mathcal{K}_{a}^{m}(G)} \leq$ $C_{m}\|v\|_{\mathcal{W}^{m, \infty}(G)}\|u\|_{\mathcal{K}_{a}^{m}(G)}$.

2.2. Graded $\kappa$-Refinement. The next step is to extend the above estimates of Proposition 2.3 to hold near the singular points. In [12], it has been shown that this can be done by considering graded meshes and the behavior of the spaces $\mathcal{K}_{a}^{m}$ under appropriate dilations. Most of the triangles in a graded mesh refinement (to be defined below) are divided into four equal triangles using the so called uniform refinement.

Definition 2.5. Let $T$ be a triangle, the uniform refinement of $T$ is to decompose $T$ as the union of four equal triangles using the midpoints of its sides. This is illustrated in Figure 2 by taking $A^{\prime}$ and $B^{\prime}$ to be located at the midpoints of $A Q$ and $B Q$, respectively, and $M$ being the midpoint of $A B$.

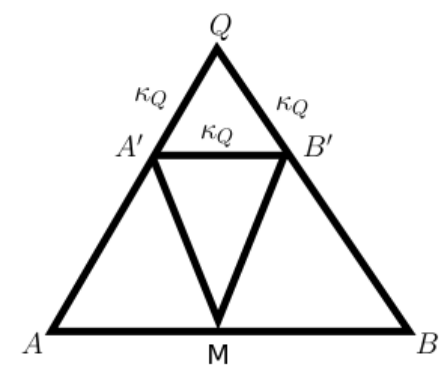

Figure 2. One refinement of the triangle $T$ with singular point $Q$, and a given $\kappa_{Q}$. When $\kappa_{Q}=\frac{1}{2}|A B|$, we have uniform refinement.

The graded mesh refinement procedure depends on some choices of parameters. Thus, for each singular point $Q$ of $\Omega$, choose a number, $\kappa_{Q} \in(0,1 / 2]$, and a set $\kappa=$ $\left\{\kappa_{Q}\right\}$. A general procedure was developed in $[12,35]$ to give a refinement pattern that obtains optimal approximation properties. We extend this construction by considering more complicated refinements of certain trapezoids that arise in this construction. To this end, for each $Q$, in addition to $\kappa_{Q}$, choose

$$
\kappa_{Q}=\kappa_{Q}^{(1)}<\kappa_{Q}^{(2)}<\ldots<\kappa_{Q}^{\left(j_{Q}\right)}=1 .
$$

The refinement is described as follows. Again, assume that the term "singular point" refers to both geometric and artificial singular points described above. Recall that the initial mesh resolves the boundary exactly and that no edge of the initial mesh contains two singular points (in the sense just described).

Definition 2.6. Let $T=Q A B$ be a triangle with a distinguished vertex $Q$. (In applications, $Q$ will be a singular point of the problem.) Then, in a $\kappa$-refinement of $T$, first divide $T$ into a smaller triangle $Q A^{\prime} B^{\prime}$ with side lengths $Q A^{\prime}=\kappa_{Q} Q A$ and $Q B^{\prime}=\kappa_{Q} Q B$, and a trapezoid, $A B B^{\prime} A^{\prime}$ with $A B$ parallel to $A^{\prime} B^{\prime}=\kappa_{Q} A B$, as shown in the left side of Figure 3 . Then, refine the trapezoid $A^{\prime} B^{\prime} B A$ by dividing the segments $Q A$ and $Q B$ with the given ratios $\kappa_{Q}^{(i)}$ introduced in Equation (18). 
More precisely, on $A A^{\prime}$, consider points $A_{1}, A_{2}, \ldots, A_{j_{Q}}$ such that $Q A_{i}=\kappa_{Q}^{(i)} Q A$ (in particular, $A_{1}=A^{\prime}$ and $A_{j_{Q}}=A$ ). Divide $Q B$ similarly and also consider the midpoint, $M$, of $A B$. Then, divide $A B B^{\prime} A^{\prime}$ into triangles by joining the corresponding points $A_{1}, A_{2}, \ldots, A_{j}, B_{1}, B_{2}, \ldots, B_{j}$, and using no point other than $M$. Thus, the resulting refinement of $Q A B$ into triangles uses only the points $Q, A, B$, $M$, and $A_{1}, A_{2}, \ldots, A_{j}, B_{1}, B_{2}, \ldots, B_{j}$.
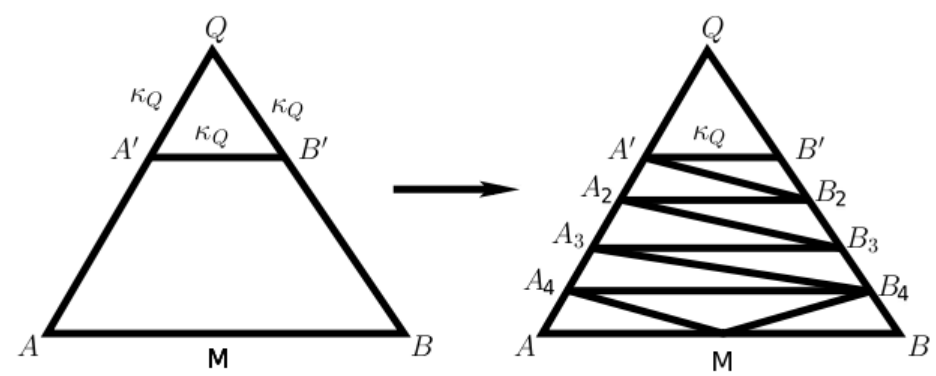

FiguRE $3 . \kappa$-refinement of triangle with singular point $Q$.

An example of a $\kappa$-refinement of a triangle $T=Q A B$ is given in the right side of Figure 3, with $j=5$. Note that if we apply the $\kappa$-refinement to two adjacent triangles that share the same distinguished point $Q$, then, the constants $\kappa_{Q}^{(i)}$ match, because they belong to the same singular point. This ensures that the resulting mesh refinement is conforming (i.e. there are no hanging nodes). See Figure 4. If further refinement is needed, the smaller triangle is refined with the same procedure described above. The trapezoid region is refined uniformly. This allows for further refinements to be done in a simple and recursive way.

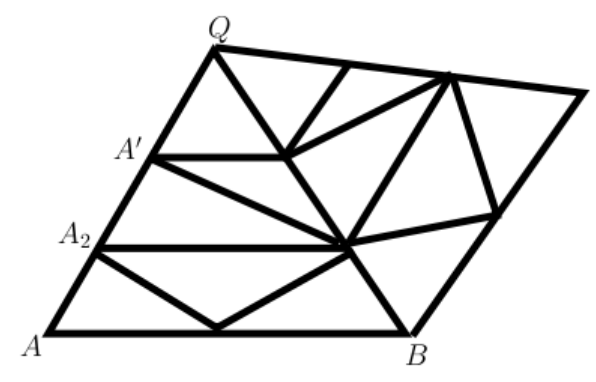

Figure 4. Matching $\kappa$-refinement for two triangles that share an edge and both touch the singular point $Q$.

Two examples of $\kappa$-refinement of a triangle are given in Figure 5. Note that a variant of the division of the bottom trapezoid is compatible with the "newest vertex bisection" method [20].

Definition 2.7. Let $\mathcal{T}$ be a mesh such that every singular point of $\Omega$ is a vertex of a triangle in $\mathcal{T}$, no triangle of $\mathcal{T}$ contains more than one singular point from among the singular points of $\Omega$, and the interface and the Dirichlet part of the boundary, 


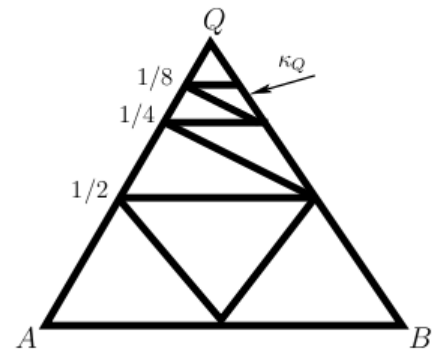

(a)

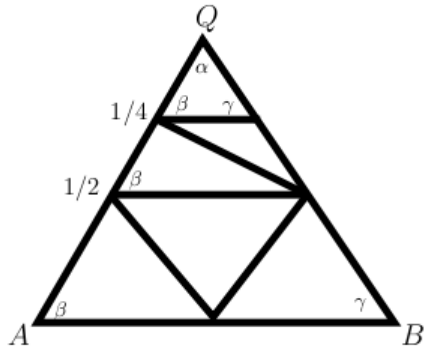

(b)

Figure 5. (a): $\kappa$-refinement of a triangle with singular point $Q$ using $\kappa_{Q} \leq 1 / 8$ and $\kappa_{Q}^{(i)}=2^{j_{Q}-i}$. (b): Similar to (a), but with one less bisection of the trapezoid. Angles are similar to angles in Figure (a).

$\partial_{D} \Omega$, is a union of edges of $\mathcal{T}$ (so $\mathcal{T}$ is aligned to the interface). A mesh with this property is called admissible. Then, define the $\kappa$-refinement of $\mathcal{T}$ to be the mesh $\kappa(\mathcal{T})$ obtained by applying uniform refinement to all triangles of $T$ that contain no singular point and by applying the $\kappa$-refinement to all triangles $T$ of $\mathcal{T}$ that contain a singular point of $\Omega$ (necessarily unique among the vertices of $T$ ). Then, the singular point of $T$ will play the role of a distinguished point in the $\kappa$-refinement of $T$.

We then have the following simple observation.

Lemma 2.8. With a fixed admissible initial mesh $\mathcal{T}_{0}, \kappa\left(\mathcal{T}_{0}\right)$ is also admissible and, hence, we can define by induction $\mathcal{T}_{n+1}=\kappa\left(\mathcal{T}_{n}\right)$.

We also obtain the following proposition stating that the minimum angle of the meshes, $\mathcal{T}_{n}$, is bounded below from 0 .

Proposition 2.9. There exists an $\alpha_{0}>0$ that only depends on the angles of the initial mesh $\mathcal{T}_{0}$ and the constants $\kappa_{Q}^{(j)}$, such that the minimum angle in any triangle in the meshes, $\mathcal{T}_{n}$, satisfies

$$
\alpha_{\min } \geq \alpha_{0} .
$$

Proof. This follows from the fact that all the triangles in the refinements, $\mathcal{T}_{n}$, belong to finitely many similarity classes, a fact easily proved by induction.

The following estimate on the number of triangles of $\mathcal{T}_{n}$ and of the dimension of the resulting finite element spaces $S_{n}=S\left(\mathcal{T}_{n}, \mathcal{F}_{n}\right)$ is useful for the optimal error estimates.

Proposition 2.10. The number of triangles, $\# \mathcal{T}_{n}$, of the mesh $\mathcal{T}_{n}$ satisfies $\# \mathcal{T}_{n} \leq$ $C 2^{2 n}$, for $C>0$. Consequently, the dimension of the finite element space, $S_{n}:=$ $S\left(\mathcal{T}_{n}, \mathcal{F}\right)$, associated to the meshes $\mathcal{T}_{n}$, satisfies $\operatorname{dim}\left(S_{n}\right) \leq C 2^{2 n}$.

Proof. The statement about $S_{n}$ follows from the corresponding statement about $\mathcal{T}_{n}$. To prove the statement about the number of elements of $\mathcal{T}_{n}, \# \mathcal{T}_{n}$, we observe that each triangle of $\mathcal{T}_{n}$ is divided in four equal triangles in $\mathcal{T}_{n+1}$, unless that triangle contains a singular point, in which case it is divided into at most $a$ triangles, where $a$ is a fixed constant. Moreover, the number of triangles of $\mathcal{T}_{n}$ that contain a singular 
point is $b$, which is a constant independent of $n$. Let $c_{n}$ be defined by $c_{0}=\# \mathcal{T}_{0}$ and $c_{n+1}=(a-4) b+4 c_{n}$. Then,

$$
c_{n}=4^{n} \# \mathcal{T}_{0}+\left(4^{n}-1\right)(a-4) b / 3 \leq C 4^{n} .
$$

Using, induction, this last inequality then gives

$$
\# \mathcal{T}_{n+1} \leq a b+4\left(\# \mathcal{T}_{n}-b\right)=(a-4) b+4 \# \mathcal{T}_{n} \leq c_{n+1} \leq C 4^{n},
$$

which is the desired estimate.

Assume that for each singular point, $Q$, a constant, $a_{Q} \in(0,1]$, is given. (Also, recall that the set of singular points includes the vertices of $\Omega$.) In applications, the constant $a_{Q}$ comes from regularity estimates, but, in general, $\kappa_{Q} \leq 2^{-m / a_{Q}}$. See for instance the discussion in Section 3. The simplest method to perform the $\kappa$-refinement, in which each triangle is divided into four smaller triangles (i.e., $j_{Q}=2$ for all $Q$ as in Figure 2), leads to smaller and smaller angles as $a_{Q} \rightarrow 0$. (Incidentally, this simple refinement is related to the ones introduced in $[2,9,12,36]$ for the Dirichlet problem.) However, this is inconvenient in some applications and has disadvantages. Thus, we present a version of the $\kappa$-refinement procedure that leads to meshes with a lower bound on the minimum angle of the triangles in the refinements, independently of $m$, as follows.

Choose $\kappa_{Q}^{(i)}=2^{j_{Q}-i}$ with $j_{Q}$ as small as possible, so that $\kappa_{Q}:=\kappa_{Q}^{(1)}=\leq 2^{-m / a_{Q}}$ in the definitions above. In particular, $\kappa_{Q}^{(1)}=2^{j_{Q}-1} \leq 2^{-m / a_{Q}}<\kappa_{Q}^{(2)}=2^{j_{Q}-2}$. The side $A Q$ of the triangle $A B Q$ in this $\kappa$-refinement is bisected until the shortest segment has length less than or equal to $\kappa_{Q}|A Q|$. Proceed similarly with $B Q$. The bottom trapezoid is refined into three triangles, whereas the intermediate trapezoids are merely bisected into two triangles. This is shown in the left side of Figure 5 . This guarantees that the minimum angle of any triangle is bounded from below by an angle independent of $m$. We now prove that the version of the $\kappa$-refinement just introduced yields this result, which may be useful for the $h p$-version of the FEM.

Theorem 2.11. Let the initial triangle $A B Q$ be refined using the $\kappa$-refinement with $\kappa_{Q}^{(i)}=2^{j_{Q}-i}$ and $\kappa_{Q}^{(1)}=\kappa_{Q} \leq 2^{-m / a_{Q}}<\kappa_{Q}^{(2)}$. Then, the minimum angle $\alpha_{\min }$ in any triangle in the meshes $\mathcal{T}_{n}$ is bounded from below,

$$
\alpha_{\min } \geq \alpha_{0}
$$

where $\alpha_{0}$ only depends on the angles of the initial mesh $\mathcal{T}_{0}$ and is independent of $m$.

Proof. A simple geometric argument shows that the trapezoids constructed from this refinement are all similar. Therefore, the smallest angle obtained is reached if one were to bisect only one trapezoid. See right side of Figure 5. The smallest angle in this configuration can be determined by the lengths of the sides and the angles of the initial triangle. Further refinement produces triangles with angles that are similar. Therefore, since $m$ only determines the number of trapezoids introduced, the minimum angle is independent of this value.

The assumption that no two singular points of $\Omega$ belong to the same triangle of the mesh is not really needed. Any reasonable division of an initial triangulation will achieve this condition. For instance, if two singular points of $\Omega$ belong to the same triangle of the mesh, then the corresponding edge can be divided into equal parts or in a ratio given by the ratio of the corresponding $\kappa$ constants. In this case, 
no new singular points are introduced on the sides of the initial triangle and this is much easier to implement. In extreme cases, however, large, skinny triangles with small angles could be introduced worsening the approximation results. In these instances, the more general procedure should be used instead. For instance, given a domain, $D$, with an initial triangulation with minimum angle $\alpha$, the refinement can now be done in a way where there is a $\alpha_{0}>0$ (but dependent on $\kappa$ and $m$ ) such that all the resulting meshes have the minimum angle greater than $\alpha_{0}$ as shown in Theorem 2.11. In either case, the following definitions and approximation results hold.

2.3. Approximation Close to the Singular Points. Again, consider a mesh, $\mathcal{T}$, and a continuous, conformally invariant family, $\mathcal{F}$, of finite elements as in Subsection 1.2. We denote by $I_{\mathcal{T}, \mathcal{F}}$ the interpolant associated to $\mathcal{T}$ and $\mathcal{F}$, as defined in Subsection 1.4. We denote by $\mathcal{T}_{n}$, the meshes on $\Omega$, and by $\mathcal{F}$, the fixed conformal invariant family of finite elements. Then, $I_{\mathcal{T}_{n}, \mathcal{F}}$ denotes the associated interpolating operator.

We now want to investigate the approximation properties afforded by the triangulation $\mathcal{T}_{n}$ close to a singular point, $Q$, of $\Omega$. Denote $V_{Q}$ to be the union of the (closed) triangles in the initial mesh $\mathcal{T}_{0}$ that have $Q$ as a singular point. Denote by $|x-y|$ the Euclidean distance from $x$ to $y$ and assume that $r_{\Omega}(x)=|x-Q|$ for $x \in V_{Q}$. By refining the initial mesh, if necessary, we may assume the closed sets $V_{Q}$ are disjoint.

For any region $G \subset V_{Q}$, denote by $\lambda G \subset V_{Q}$ the region obtained by dilating $G$ with respect to $Q$ with ratio $\lambda<1$. The following interpolation estimates are then similar to those in $[12,35]$, but deal with a higher order approximation. In particular, we need the following simple lemma that is proved by a direct calculation in [12]. Recall the definitions of the norms \|\|$_{\mathcal{K}_{a}^{m}(G)}=\|\|_{\mathcal{K}_{a}^{m}\left(G ; r_{\Omega}\right)}$ and \|\|$_{\mathcal{W}^{m, \infty}(G)}=\|\|_{\mathcal{W}^{m, \infty}\left(G ; r_{\Omega}\right)}$, Equations (15) and (17).

Lemma 2.12. Let $Q$ be a singular point of $\Omega$ and $G \subset V_{Q} \subset \bar{\Omega}$ be an open set. Denote $G^{\prime}=\lambda G, 0<\lambda<1$, and $u_{\lambda}(x):=u(\lambda x)$, then

$$
\left\|u_{\lambda}\right\|_{\mathcal{K}_{a}^{m}(G)}=\lambda^{a-1}\|u\|_{\mathcal{K}_{a}^{m}\left(G^{\prime}\right)} \quad \text { and } \quad\left\|u_{\lambda}\right\|_{\mathcal{W}^{m, \infty}(G)}=\|u\|_{\mathcal{W}^{m, \infty}\left(G^{\prime}\right)} .
$$

We now define the space of functions to be approximated. We proceed as in [35]. Let $\chi_{Q}$ be a smooth function that is equal to 1 near each singular point $Q \in \mathcal{V}$. We assume that the functions, $\chi_{Q}$, have disjoint supports and, in case $Q$ is an interior point of the domain, then $\chi_{Q}$ vanishes close to the boundary of $\Omega$. If $\chi_{Q}$ is supported near a vertex of $\Omega$, then we assume that it satisfies the Neumann boundary conditions on each adjacent side. If the diffusion matrix, $A$, is scalar, then we can take $\chi_{Q}$ to be a function of the distance function to the point $Q$. Denote by $\mathcal{W} \subset \mathcal{V}$, the subset of the set $\mathcal{V}$ of singular points of the problem that are either a vertex whose adjacent sides have Neumann conditions (a so called "Neumann-Neumann vertex"), or a non-smooth interface point interior to $\Omega$, or a point where the interface touches the interior of $\partial_{N} \Omega$. Thus, $\mathcal{W}$ includes the points that belong to more than two of the subdomains, $\bar{\Omega}_{j}$ (the so called multiple junction points), which are typically interior points of $\Omega$. We also have that $\mathcal{W}$ is the set of points $Q \in \mathcal{V}$ such that $\chi_{Q}$ satisfies the boundary conditions of the problem. Then, we define $W_{s}$ to be the linear span of the functions $\chi_{Q}$, and, hence, all the functions in $W_{s}$ satisfy the boundary conditions of the problem. We also need to 
introduce the broken Sobolev spaces $\hat{\mathcal{K}}_{a}^{m}(\Omega)$, defined in terms of the decomposition $\bar{\Omega}=\cup_{j=1}^{K} \bar{\Omega}_{j}$,

$$
\hat{\mathcal{K}}_{a}^{m}(\Omega):=\left\{u: \Omega \rightarrow \mathbb{R},\left.u\right|_{\Omega_{j}} \in \mathcal{K}_{a}^{m}\left(\Omega_{j}\right), j=1, \ldots, K\right\} .
$$

We then let the approximation space be

$$
V:=\hat{\mathcal{K}}_{a+b+1}^{m+1}(\Omega) \cap \mathcal{K}_{a+b+1}^{1}(\Omega)+W_{s},
$$

for the fixed approximation parameter $m \geq 1$ and some parameters $a>0, a+b>0$. This choice of approximation space is suggested by the regularity results of [35], which state that the solution, $u$, of the transmission/boundary value problem (1) is such that $u \in V$ for $b=0$ (see [34] for some related results). The additional parameter $b$ satisfying $a+b>0$ is introduced with some applications using the least squares finite-element method in mind [32]. Notice that for each triangle $T \in \mathcal{T}_{n}$, we have $T \subset \Omega_{j}$, since the initial mesh $\mathcal{T}_{0}$ is aligned with the interface and, hence, $u \in \mathcal{K}_{a+b+1}^{m+1}(T)$. Thus, we work with the broken weighted Sobolev spaces in the same way we would work with the usual weighted Sobolev spaces.

The norm on $V$ is given by the direct sum norm for any choice of a norm \|\|$_{W_{s}}$ on the finite-dimensional space $W_{s}$ :

$$
\left\|u_{0}+w_{s}\right\|_{V}=\left\|u_{0}\right\|_{\mathcal{K}_{a+b+1}^{1}(\Omega)}+\sum_{j=1}^{K}\left\|u_{0}\right\|_{\mathcal{K}_{a+b+1}^{m+1}\left(\Omega_{j}\right)}+\left\|w_{s}\right\|_{W_{s}},
$$

where $u_{0} \in \hat{\mathcal{K}}_{a+b+1}^{m+1}(\Omega) \cap \mathcal{K}_{a+b+1}^{1}(\Omega)$ and where $w_{s}$ is always an element in $W_{s}$. Then, we have the following lemma (which remains valid in three dimensions). For definiteness, we choose $\left\|\sum_{Q} a_{Q} \chi_{Q}\right\|_{W_{s}}=\sum_{Q}\left|a_{Q}\right|$. Also, notice that $\mathcal{K}_{a+b+1}^{1}(\Omega) \cap$ $\hat{\mathcal{K}}_{a+b+1}^{m+1}(\Omega)$ is a closed subspace of $\hat{\mathcal{K}}_{a+b+1}^{m+1}(\Omega)$ for $m \geq 1$, and, hence, the term $\left\|u_{0}\right\|_{\mathcal{K}_{a+b+1}^{1}(\Omega)}$ is not really necessary in the definition of the $V$-norm. Another good choice of a norm on $W_{s}$ is the restriction of any Sobolev norm $H^{k}$ on $\Omega$ to $W_{s}$.

The following lemma relies essentially on the additional condition that $u_{0} \in$ $\mathcal{K}_{a+b+1}^{1}(\Omega)$, which enforces the continuity across the interface(s).

Lemma 2.13. There is a continuous embedding $V \rightarrow \mathcal{C}(\bar{\Omega})$. In particular, for each singular point $Q \in \mathcal{V}$ and for each $u \in V$, the value $u(Q)$ is well defined and depends continuously on $u$.

Proof. Let $u \in V$ and $U \subset \Omega$ be an open subset at positive distance from the set of singular points $\mathcal{V}$. Then, $u \in \mathcal{C}(\bar{\Omega})$, since $m+1 \geq 2>d / 2$ and, hence, $H^{m+1}(\Omega) \subset \mathcal{C}(\bar{\Omega})$, by the Sobolev embedding theorem. By the dilation invariance of the spaces $\mathcal{K}_{a}^{m}\left(\Omega_{j}\right)$, Lemma 2.12 ,

$$
\mathcal{K}_{1}^{m+1}\left(\Omega_{j}\right) \subset \mathcal{C}\left(\overline{\Omega_{j}} \backslash \mathcal{V}\right) \cap L^{\infty}\left(\Omega_{j}\right)
$$

Then, notice that any function $u \in \mathcal{K}_{a+b+1}^{1}(\Omega)$ whose restriction to each $\Omega_{j}$ extends to a continuous function on $\overline{\Omega_{j}} \backslash \mathcal{V}$ is actually continuous on $\bar{\Omega} \backslash \mathcal{V}$. This gives

$$
\hat{\mathcal{K}}_{a+b+1}^{m+1}(\Omega) \cap \mathcal{K}_{a+b+1}^{1}(\Omega)=r_{\Omega}^{a+b}\left(\hat{\mathcal{K}}_{1}^{m+1}(\Omega) \cap \mathcal{K}_{a+b+1}^{1}(\Omega)\right) \subset \mathcal{C}(\bar{\Omega}),
$$

as stated, since $a+b>0$. 
It follows that any $u \in V:=\hat{\mathcal{K}}_{a+b+1}^{m+1}(\Omega) \cap \mathcal{K}_{a+b+1}^{1}(\Omega)+W_{s}$ can be written as (22) $u=u_{0}+w_{s}, \quad w_{s}:=\sum_{Q \in \mathcal{W}} u(Q) \chi_{Q} \in W_{s}$ and $u_{0} \in \hat{\mathcal{K}}_{a+b+1}^{m+1}(\Omega) \cap \mathcal{K}_{a+b+1}^{1}(\Omega)$, and we call this decomposition the canonical decomposition of $u \in V$.

Assumptions: For simplicity, we assume from now on that the constants $\kappa_{Q}$ are all the same and we let $\kappa=\kappa_{Q}$. We also assume that $\kappa \leq 2^{-m / a}$, for some fixed $a>0$. Also, recall that the approximation degree $m \geq 1$ is fixed.

Now estimate the interpolation error on the triangles of the mesh $\mathcal{T}_{n}$ that are close to a singular point. More precisely, for each singular point $Q \in \mathcal{V}$, define $V_{Q}^{(n)}$ to be the union of all the closures of triangles $T \in \mathcal{T}_{n}$ that have $Q$ as a vertex. Similarly, define $U_{Q}^{(n)}$ to be the union of all the closures of triangles $T \in \mathcal{T}_{n}$ that touch (i.e., intersect) $V_{Q}^{(n)}$. Then, choose functions $\eta_{Q}^{(n)} \in \mathcal{C}^{\infty}(\bar{\Omega})$ that are equal to 0 on $V_{Q}^{(n)}$ and are equal to 1 outside $U_{Q}^{(n)}$. These functions, $\eta_{Q}^{(n)}$, can be chosen such that they correspond to each other with respect to dilations centered at $Q$, in an obvious sense. Thus, for each $n, \eta_{Q}^{(n)}$ is obtained by a dilation with ratio $\kappa^{n-1}$ from $\eta_{Q}^{(1)}$.

Recall that we have assumed that $\kappa \leq 2^{-m / a}, a>0$. We then have the following simple lemma.

Lemma 2.14. Let $G \subset \Omega$ be an open subset. Using the functions $\eta_{Q}^{(n)}$ introduced above, there exists a constant $C>0$ such that

$$
\left\|\left(1-\eta_{Q}^{(n)}\right) u\right\|_{\mathcal{K}_{b+1}^{1}(G)} \leq C 2^{-m n}\|u\|_{\mathcal{K}_{a+b+1}^{m+1}(G)},
$$

for any $u \in \mathcal{K}_{a+b+1}^{m+1}\left(G, r_{\Omega}\right)$.

Proof. Assume $G=\Omega$, for simplicity of notation. The general case is identical. The support of $\left(1-\eta_{Q}^{(n)}\right) u$ is contained in a set on which $r_{\Omega} \leq C \kappa^{n}$, with $C$ independent of $Q$ or $n$ and, hence,

$$
\left\|\left(1-\eta_{Q}^{(n)}\right) u\right\|_{\mathcal{K}_{b+1}^{1}} \leq C\left(\kappa^{n}\right)^{a}\left\|\left(1-\eta_{Q}^{(n)}\right) u\right\|_{\mathcal{K}_{a+b+1}^{1}} \leq C 2^{-m n}\|u\|_{\mathcal{K}_{a+b+1}^{m+1}},
$$

where in the last inequality, Lemma 2.4 is used as well as the fact that the norms $\left\|1-\eta_{Q}^{(n)}\right\|_{\mathcal{W}^{m, \infty}}$ are independent of $n$ by the dilation invariance of the $\mathcal{W}^{m, \infty}$ norm (Lemma 2.12).

To continue the study of the error estimates, now assume that, for each fixed $n$, all the functions $1-\eta_{Q}^{(n)}$ have disjoint supports, so $\left(1-\eta_{Q}^{(n)}\right)\left(1-\eta_{Q^{\prime}}^{(n)}\right)=0$ for $Q \neq Q^{\prime} \in \mathcal{V}$ and, hence, $\eta^{(n)}:=\prod_{Q \in \mathcal{V}} \eta_{Q}^{(n)}$ satisfies $1=\eta^{(n)}+\sum_{Q}\left(1-\eta_{Q}^{(n)}\right)$. Denote then by

$$
\tilde{u}_{n}=\eta^{(n)} u+\sum_{Q \in \mathcal{V}} u(Q)\left(1-\eta_{Q}^{(n)}\right)=\eta^{(n)} u+\sum_{Q \in \mathcal{W}} u(Q)\left(1-\eta_{Q}^{(n)}\right),
$$

for $u \in V:=\mathcal{K}_{a+b+1}^{m+1}(\Omega)+W_{s}$. Note that $\tilde{u}_{n}$ is well defined since $V$ consists of continuous functions (Lemma 2.13).

Lemma 2.14 then yields the following corollary. 
Corollary 2.15. Using the notation $\tilde{u}_{n}$ of Equation (24), we have that there exists a constant $C>0$, independent of $n$, such that

$$
\left\|u-\tilde{u}_{n}\right\|_{\mathcal{K}_{b+1}^{1}(\Omega)} \leq C 2^{-m n}\|u\|_{V}
$$

for any $u \in V:=\hat{\mathcal{K}}_{a+b+1}^{m+1}(\Omega) \cap \mathcal{K}_{a+b+1}^{1}(\Omega)+W_{s}$, with canonical decomposition $u=u_{0}+w_{s}, u_{0} \in \mathcal{K}_{a+b+1}^{m+1}(\Omega) \cap \mathcal{K}_{a+b+1}^{1}(\Omega)$ and $w_{s} \in W_{s}$.

Proof. The proof is obtained by estimating on each region, $\Omega_{j}$, so we assume that $K=1$ (that is, there are no interfaces). Here, $u=u_{0}+\sum_{Q} u(Q) \chi_{Q}$, with $u_{0} \in$ $\mathcal{K}_{a+b+1}^{m+1}(\Omega)$, by the definition of the approximation space $V$. Since $1=\eta^{(n)}+$ $\sum_{Q}\left(1-\eta_{Q}^{(n)}\right)$, we have

$$
u-\tilde{u}_{n}=\sum_{Q \in \mathcal{W}}(u-u(Q))\left(1-\eta_{Q}^{(n)}\right) .
$$

Since $\left(1-\chi_{Q}\right)\left(1-\eta_{Q}^{(n)}\right)$ is a smooth function on $\bar{\Omega}$ that is zero near the set $\mathcal{V}$ of singular points, we have $\left(1-\chi_{Q}\right)\left(1-\eta_{Q}^{(n)}\right) \in \mathcal{K}_{a+b+1}^{m+1}(\Omega)$ for all $m$ and $a$ and $b$ with $a>0, a+b>0$.

By decreasing supports of the functions $\eta_{Q}^{(n)}$, if necessary, we may assume that $\chi_{Q^{\prime}}\left(1-\eta_{Q}^{(n)}\right)=0$ for $Q \neq Q^{\prime}$ and that $\left(1-\chi_{Q}\right)\left(1-\eta_{Q}^{(n)}\right)=0$. Write $u \in V$ as $u=u_{0}+\sum_{Q \in \mathcal{W}} u(Q) \chi_{Q}$, with $u_{0} \in \mathcal{K}_{a+b+1}^{m+1}(\Omega)$. Equation (25) then gives

$$
u-\tilde{u}_{n}=\sum_{Q \in \mathcal{W}} u_{0}\left(1-\eta_{Q}^{(n)}\right) .
$$

The result then follows from Lemma 2.14.

We also mention the following corollary of the above proof.

Corollary 2.16. Using the notation of Corollary 2.15, if $u \in W_{s}$, then $u=\tilde{u}_{n}$.

Proof. Indeed, under the assumptions of this corollary, $u_{0}=0$ and, hence, the result is given by Equation (26).

We now introduce the modified interpolation operator by

$$
u_{I, n}=I_{\mathcal{T}_{n}, \mathcal{F}}\left(\tilde{u}_{n}\right) \in S_{n}:=S\left(\mathcal{T}_{n}, \mathcal{F}\right),
$$

with $\tilde{u}_{n}$ defined in Equation (24). Note that the modified interpolation operator allows the smoothness properties of $u$ close to the singular points to be ignored, since $\tilde{u}_{n}$ (unlike $u$ ) is constant close to each singular point. Thus, only smoothness estimates on $u$ away from the singular points are needed, where they are the same as the classical estimates, (an example is the Sobolev embedding $H^{2} \subset \mathcal{C}$, valid in two and three dimensions).

We want to approximate functions, $u$ in $V:=\mathcal{K}_{a+b+1}^{m+1}(\Omega)+W_{s}$, and the space introduced in Equation (20). We first show that we assume $u \in \mathcal{K}_{a+b+1}^{m+1}(\Omega)$.

Let $T \in \mathcal{T}_{0}$ be a triangle that has a vertex that is a singular point $Q$ of $\Omega$. Let $\kappa^{n} T \in \mathcal{T}_{n}$ be the triangle obtained by dilating $T$ with ratio $\kappa^{n}=\kappa_{Q}^{n}$ and center $Q$, that is, the triangle that is similar to $T$ with ratio $\kappa^{n}$, has $Q$ as a vertex, and has all sides parallel to the sides of $T$. Then, $\kappa^{n} T \subset \kappa^{n-1} T$ for $n \geq 1$ and $\kappa^{n} T \in \mathcal{T}_{n}$. We assume that for all triangles $T \in \mathcal{T}_{0}$ with singular vertex $Q \in \mathcal{V}$, we have $\chi_{Q}=1$ on $\kappa T$, for all $Q$. 
Lemma 2.17. Let $w=\sum_{Q} a_{Q} \chi_{Q} \in W_{s}$. Then,

$$
\left\|w-w_{I, n}\right\|_{\mathcal{K}_{b+1}^{1}(\Omega)} \leq C 2^{-m n} \sum_{Q}\left|a_{Q}\right|=: C 2^{-m n}\|w\|_{W_{s}}=: C 2^{-m n}\|w\|_{V},
$$

for a constant $C$ that is independent of $w$ and $n$.

Proof. Let $W=\Omega \backslash \cup \kappa T$, where the union is over all triangles $T \in \mathcal{T}_{0}$ that have a vertex in the set $\mathcal{V}$ of singular points of the problem. Then, $w=w_{I, n}$ outside $W$, by Corollary 2.16. The result is then a consequence of Proposition 2.3 and of the fact that $h \leq C 2^{-n}$ for the mesh $\mathcal{T}_{n}$, with $C$ a constant depending only on the initial mesh $\mathcal{T}_{0}$.

We want to extend the above lemma to $u \in V$. By linearity, we assume that $u \in \mathcal{K}_{a+b+1}^{m+1}(\Omega)$, using the above lemma. The estimates are obtained by breaking them into regions. We begin with the regions closest to the singularities.

Lemma 2.18. Denote by $\kappa^{n} T \subset T \subset \Omega_{j}$ the triangle with singular point, $Q$, obtained from $T \in \mathcal{T}_{0}$ after $n$ refinements. Let $u \in \hat{\mathcal{K}}_{a+b+1}^{m+1}(\Omega) \cap \mathcal{K}_{a+b+1}^{1}(\Omega)$. Then,

$$
\left\|u-u_{I, n}\right\|_{\mathcal{K}_{b+1}^{1}\left(\kappa^{n} T\right)} \leq C 2^{-m n}\|u\|_{\mathcal{K}_{a+b+1}^{m+1}\left(\kappa^{n} T\right)},
$$

where $C$ depends on $m$ and $\kappa$, but not on $n$ or $T$. Here, $u_{I, n}$ is the modified interpolant given by Equation (27).

Proof. Since $\tilde{u}_{n}=u(Q)=0$ on $\kappa^{n} T, u_{I, n}=I_{\mathcal{T}_{n}, \mathcal{F}}\left(\tilde{u}_{n}\right)=u(Q)=0$ on $\kappa^{n} T$ as well. Then,

$$
\left\|u-u_{I, n}\right\|_{\mathcal{K}_{b+1}^{1}\left(\kappa^{n} T\right)}=\|u\|_{\mathcal{K}_{b+1}^{1}\left(\kappa^{n} T\right)} \leq C 2^{-m n}\left\|u_{0}\right\|_{\mathcal{K}_{a+b+1}^{m+1}\left(\kappa^{n} T\right)},
$$

by Lemma 2.14 .

The bounds on $\kappa^{n} T$ of the previous lemma are combined with bounds on sets of the form $\kappa^{j} T \backslash \kappa^{j+1} T$ to obtain the following estimate on the arbitrary, but fixed, triangle $T \in \mathcal{T}_{0}$ that has a vertex $Q$ that is a singular point of $\Omega$. Recall that $\lambda G$ is obtained from $G$ by dilating with ratio $\lambda<1$ and center $Q$.

Proposition 2.19. Consider the triangles $\kappa \xi T \subset \xi T \subset \Omega_{j}$, where $T$ is a triangle with one vertex $Q \in \mathcal{V}$, a singular point of $\Omega$. Let $\mathcal{T}=\left(T_{j}\right)$ be a triangulation of $G:=\xi T \backslash \kappa \xi T$ with angles $\geq \alpha$ and edges $\leq h$. Let $u \in \mathcal{K}_{a+b+1}^{m+1}\left(\Omega_{j}\right)$. Then, the interpolant $I_{\mathcal{T}, \mathcal{F}}(u) \in S(\mathcal{T}, \mathcal{F})$ satisfies

$$
\left\|u-I_{\mathcal{T}, \mathcal{F}}(u)\right\|_{\mathcal{K}_{b+1}^{1}\left(G ; r_{\Omega}\right)} \leq C(a, \kappa, \alpha, m) \xi^{a}(h / \xi)^{m}\|u\|_{\mathcal{K}_{a+b+1}^{m+1}\left(G ; r_{\Omega}\right)},
$$

with $C(a, \kappa, \alpha, m)$ independent of $\xi, h$, and $u$.

Proof. Let $\tilde{\ell}$ be the distance from $Q$ to the opposite side of $T$. Assume that $Q$ is the origin, to simplify the notation, and recall the dilation function $u_{\lambda}$, where $u_{\lambda}(x)=u(\lambda x), x \in \mathbb{R}^{2}$. Also, recall that the dilation commutes with interpolation by the assumption that the family of finite elements, $\mathcal{F}$, is conformally invariant. Using Lemma 2.12 with $\lambda=\xi$, Proposition 2.3 is applied to the region $G^{\prime}=T \backslash$ $\kappa T=\lambda^{-1} G \subset \Omega$. Denoting by $M=C(\alpha, m, \kappa \tilde{\ell} \xi, a, 1)$ the constant of Proposition 2.3 , we obtain

$$
\begin{aligned}
\left\|u-I_{\mathcal{T}, \mathcal{F}}(u)\right\|_{\mathcal{K}_{b+1}^{1}(G)} & =\left\|u_{\lambda}-I_{\mathcal{T}, \mathcal{F}}(u)_{\lambda}\right\|_{\mathcal{K}_{b+1}^{1}\left(G^{\prime}\right)}=\left\|u_{\lambda}-I_{\mathcal{T}, \mathcal{F}}\left(u_{\lambda}\right)\right\|_{\mathcal{K}_{b+1}^{1}\left(G^{\prime}\right)} \\
& \leq M(h / \xi)^{m}\left\|u_{\lambda}\right\|_{\mathcal{K}_{a+b+1}^{m+1}\left(G^{\prime}\right)}=M \xi^{a}(h / \xi)^{m}\|u\|_{\mathcal{K}_{a+b+1}^{m+1}(G)} .
\end{aligned}
$$


This completes the proof.

Next, denote $u_{I, n}$ to be the modified interpolant of Equation (27).

Proposition 2.20. Let $T \in \mathcal{T}_{0}$ have the singular point $Q$ as a vertex. Then, there exists a constant $C>0$, such that

$$
\left\|u-u_{I, n}\right\|_{\mathcal{K}_{b+1}^{1}(T)} \leq C 2^{-m n}\|u\|_{V}=C 2^{-m n}\left(\left\|u_{0}\right\|_{\mathcal{K}_{a+b+1}^{m+1}(T)}+\left\|w_{s}\right\|_{W_{s}}\right),
$$

for all $n$ and all $u \in V$ with canonical decomposition $u=u_{0}+w_{s}$.

Proof. Assume that $w_{s}=0$, by Lemma 2.17. Also, notice that $T \subset \Omega_{j}$, since the initial mesh $\mathcal{T}_{0}$ is aligned with the interface and, hence, $u \in \mathcal{K}_{a+b+1}^{m+1}(T)$. Fix $n$. The proof of the proposition follows from the estimates on the subsets $\kappa^{j-1} T \backslash \kappa^{j} T$, $1 \leq j \leq n$, (Proposition 2.19) and from the estimate on $\kappa^{n} T$ (Lemma 2.18). Let $\tilde{u}_{n}$ be as defined in Equation (24). Recall that we write $\mathcal{K}_{a}^{m}\left(G ; r_{\Omega}\right)=\mathcal{K}_{a}^{m}(G)$ when convenient. In view of Corollary 2.15 , it is enough to show that

$$
\left\|\tilde{u}_{n}-u_{I, n}\right\|_{\mathcal{K}_{b+1}^{1}(T)} \leq C 2^{-m n}\|u\|_{V}=C 2^{-m n}\left(\left\|u_{0}\right\|_{\mathcal{K}_{a+b+1}^{m+1}(T)}+\left\|w_{s}\right\|_{W_{s}}\right),
$$

with $C$ a possibly different constant. Then, write

$$
\left\|\tilde{u}_{n}-u_{I, n}\right\|_{\mathcal{K}_{b+1}^{1}(T)}^{2}=\left\|\tilde{u}_{n}-u_{I, n}\right\|_{\mathcal{K}_{b+1}^{1}\left(\kappa^{n} T\right)}^{2}+\sum_{j=1}^{n}\left\|\tilde{u}_{n}-u_{I, n}\right\|_{\mathcal{K}_{b+1}^{1}\left(\kappa^{j-1} T \backslash \kappa^{j}\right)}^{2} .
$$

Recall that $u_{I, n}=I_{\mathcal{T}_{n}, \mathcal{F}}\left(\tilde{u}_{n}\right) \in S_{n}:=S\left(\mathcal{T}_{n}, \mathcal{F}\right)$ and that $\tilde{u}_{n}=0$ on $\kappa^{n} T$. The first term $\left\|\tilde{u}_{n}-u_{I, n}\right\|_{\mathcal{K}_{b+1}^{1}\left(\kappa^{n} T\right)}^{2}$ is, thus, zero. Definition 2.7 shows that the mesh size $h$ of the restriction of the mesh $\mathcal{T}_{n}$ to $\kappa^{j-1} T \backslash \kappa^{j} T$, is $\leq C \kappa^{j-1} 2^{j-1-n}$, for a constant $C$ that depends only on $\mathcal{T}_{0}$. Let $G=\kappa^{j-1} T \backslash \kappa^{j} T$. Then, using the notation in Proposition 2.19, we have that $\xi=\kappa^{j-1}$ and, therefore,

$$
\begin{gathered}
\left\|\tilde{u}_{n}-u_{I, n}\right\|_{\mathcal{K}_{b+1}^{1}(G)} \leq C_{1} \kappa^{(j-1) a}\left(\kappa^{j-1} 2^{j-1-n} / \kappa^{j-1}\right)^{m}\left\|\tilde{u}_{n}\right\|_{\mathcal{K}_{a+b+1}^{m+1}(G)} \\
\leq C_{2} 2^{-(j-1) m} 2^{-n m+(j-1) m}\left\|\tilde{u}_{n}\right\|_{\mathcal{K}_{a+b+1}^{m+1}(G)}=C_{2} 2^{-n m}\left\|\tilde{u}_{n}\right\|_{\mathcal{K}_{a+b+1}^{m+1}(G)} \\
\leq C_{3} 2^{-n m}\left\|\tilde{u}_{n}\right\|_{\mathcal{K}_{a+b+1}^{m+1}(G)},
\end{gathered}
$$

where $C_{1}, C_{2}$, and $C_{3}$ depend on $\kappa$, but not on $u, n$, or $j$. The last inequality is from Lemma 2.14. We complete the proof by adding up all the above error estimates on the subsets $G:=\kappa^{j-1} T \backslash \kappa^{j} T, 1 \leq j \leq n$.

Consider now the set $G=\Omega \backslash\left(\cup_{Q \in \mathcal{V}} \cup_{Q \in T \in \mathcal{T}_{0}} \bar{T}\right)$, that is, the set $G$ is obtained by removing from $\Omega$ all the triangles of the initial mesh $\mathcal{T}_{0}$ that have a vertex among the singular points of the problem. We write by abuse of notation, $Q \in T$, when we really mean that the vertex $Q$ of $T$ is in the closure of $T$. We then have the following main approximation result.

Theorem 2.21. Assume $u=u_{0}+w_{s} \in V$, where $u_{0} \in \mathcal{K}_{a+b+1}^{1}(\Omega),\left.u\right|_{\Omega_{j}} \in$ $\mathcal{K}_{a+b+1}^{m+1}\left(\Omega_{j}\right)$, and $w_{s}=\sum_{Q \in \mathcal{W}} a_{Q} \chi_{Q} \in W_{s}$. Assume $a>0, a+b>0$, and $\kappa \leq 2^{-m / a}$. Let $\mathcal{T}_{n}$ be the $n$-th refinement of an initial triangulation, $\mathcal{T}_{0}$, aligned with the interface. Let $S_{n}:=S_{n}\left(\mathcal{T}_{n}, \mathcal{F}\right)$ be the associated finite-element space given in equation (9) and let $u_{I, n} \in S_{n}$ be the modified interpolant associated to $\mathcal{T}_{n}$ and $\mathcal{F}$, Equation (27). Then, there exists $C>0$, independent of $n$ or $u$, such that

$$
\left\|u-u_{I, n}\right\|_{\mathcal{K}_{b+1}^{1}(\Omega)} \leq C 2^{-m n}\left(\sum_{j}\|u\|_{\mathcal{K}_{a+b+1}^{m+1}\left(\Omega_{j}\right)}+\sum_{Q}\left|a_{Q}\right|\right)=: C 2^{-m n}\|u\|_{V} .
$$


Note that while $u \in V:=\hat{\mathcal{K}}_{a+b+1}^{m+1}(\Omega) \cap \mathcal{K}_{a+b+1}^{1}(\Omega)+W_{s}$, the difference $u-u_{I, n} \in$ $\mathcal{K}_{a+b+1}^{m+1}(\Omega)$.

Proof. Again, assume that $w_{s}=0$, by Lemma 2.17. The proof is then an immediate consequence of the estimates in Propositions 2.20 and 2.3 applied, respectively, to the triangles $T \in \mathcal{T}_{0}$ that have singular points as vertices and to the region $W$ that is the complement of these triangles in $\Omega$ (as defined above). We then have

$$
\begin{aligned}
& \left\|u-u_{I, n}\right\|_{\mathcal{K}_{b+1}^{1}(\Omega)}^{2}=\sum_{Q \in \mathcal{V}} \sum_{Q \in T}\left\|u-u_{I, n}\right\|_{\mathcal{K}_{b+1}^{1}(T)}^{2}+\left\|u-u_{I, n}\right\|_{\mathcal{K}_{b+1}^{1}(W)}^{2} \\
& \quad \leq C 2^{-m n}\left(\sum_{Q \in \mathcal{V}} \sum_{Q \in T}\|u\|_{\mathcal{K}_{a+b+1}^{m+1}(T)}^{2}+\sum_{j=1}^{K}\|u\|_{\mathcal{K}_{a+b+1}^{m+1}\left(W \cap \Omega_{j}\right)}^{2}\right) \leq C 2^{-m n}\|u\|_{V} .
\end{aligned}
$$

The proof is now complete.

\section{Applications to the Finite-Element Method}

In this section, we apply the results of the previous sections to obtain quasioptimal convergence rates for the finite-element solution of a transmission/interface problem, such as (1), using the meshes $\mathcal{T}_{n}$.

Recall that $\bar{\Omega}=\cup_{j=1}^{K} \bar{\Omega}_{j}$, where $\Omega_{j}$ are disjoint polygonal domains. Let $\Gamma:=$ $\partial \Omega \backslash \cup_{j=1}^{K} \partial \Omega_{k}$ denote the interface. Assume the coefficients $A=\left[a_{i j}\right]$ have only jump discontinuities across the interface $\Gamma$. We are interested in approximating the solution of the boundary value/interface problem, (1), stated in the Introduction. Note that this problem is really formulated in a weak sense, which implies the usual matching and jump conditions at the interface,

$$
u_{+}=u_{-}, \quad D_{\nu+}^{A} u=D_{\nu-}^{A} u
$$

where we have labeled the non-tangential limits $u_{+}, u_{-}$of $u$ at each side of the interface, and denote the respective conormal derivatives, $D_{\nu+}^{A}$ and $D_{\nu-}^{A}$, by $D_{\nu \pm}^{A} u:=$ $\sum_{i, j} \nu_{i} A^{i, j} \partial_{j} u_{ \pm}=\nu \cdot A \cdot \nabla u_{ \pm}$, where $\nu$ is a choice of unit normal vector to the interface $\Gamma$. We shall also assume that $-\operatorname{div}(A \nabla)$ is uniformly strongly elliptic, in the usual sense, that is, we assume that there exists $C>0$ such that

$$
\sum_{i, j=1}^{2} A^{i, j}(x) \xi_{i} \xi_{j} \geq C\left(\xi_{1}^{2}+\xi_{2}^{2}\right)
$$

for all $x \in \Omega$ and $\xi \in \mathbb{R}^{2}$. Then, one of the main results of [35] states that the solution, $u$, of the boundary value/interface problem (1) is such that $u \in V$, where $V$ is the approximation spaces introduced in Equation (20) with $b=0$. More precisely, we have the following result.

Theorem 3.1. Assume $\partial_{D} \Omega \neq \emptyset$ and that $\Omega$ is connected, then there exists $\eta>0$ with the following property. Assume $0<a<\eta, m \in \mathbb{Z}_{+}$, and $f \in \hat{\mathcal{K}}_{a-1}^{m-1}(\Omega)$, then there exists a unique solution $u=u_{0}+w_{s}, y_{0} \in \hat{\mathcal{K}}_{a+1}^{m+1}(\Omega) \cap \mathcal{K}_{a+1}^{1}(\Omega), w_{s} \in W_{s}$, of Equation (1) (the transmission problem). Moreover, there is a constant $C>0$ such that

$$
\|u\|_{V}:=\left\|u_{0}\right\|_{\mathcal{K}_{a+1}^{1}(\Omega)}+\sum_{j=1}^{K}\left\|u_{0}\right\|_{\mathcal{K}_{a+1}^{m+1}\left(\Omega_{j}\right)}+\left\|w_{s}\right\|_{W_{s}} \leq C \sum_{j=1}^{K}\|f\|_{\mathcal{K}_{a-1}^{m-1}\left(\Omega_{j}\right)},
$$


The constant $C$ depends on $\Omega, m, a$, and $A^{i j}$, but not on $f$. The solution u also satisfies the matching interface conditions (30).

In the case of the pure Neumann problem we have to take into account the nonuniqueness of the solution. Once we do that, a similar statement holds true.

The bilinear form associated to the problem $(1)$ is $B(u, v)=\int_{\Omega}(\nabla u) \cdot A \cdot(\nabla v) d x$, as usual. Then, $u_{n} \in S_{n}=S\left(\mathcal{T}_{n}, \mathcal{F}_{n}\right)$ denotes the Galerkin approximation of the solution $u$ of problem (1), namely, it is the unique $u_{n} \in S_{n}$ satisfying

$$
B\left(u_{n}, v_{n}\right)=\left(f, v_{n}\right), \quad \forall v_{n} \in S_{n} .
$$

Recall the norm \|\|$_{V}$ introduced in Equation (21), defined on the spaces $V:=$ $\hat{\mathcal{K}}_{a+1}^{m+1}(\Omega) \cap \mathcal{K}_{a+1}^{1}(\Omega)+W_{s}$. Then, we have the following theorem,

Theorem 3.2. Let $m \geq 1$, assume the Dirichlet part of the boundary is not empty, and let $u$ be the corresponding solution to problem (1) with $f \in \hat{\mathcal{K}}_{a-1}^{m-1}(\Omega)$ for all $j$, where $0<a<\eta$, with $\eta$ as in Theorem 3.1. Let $\mathcal{T}_{n}$ be the $n$-th $\kappa$-refinement of an initial triangulation $\mathcal{T}_{0}$ as in Definition 2.7, let $S_{n}:=S_{n}\left(\mathcal{T}_{n}, m\right)$ be the associated finite element space given in equation (9) and let $u_{n}=u_{S_{n}} \in S_{n}$ be the finite element solution defined in (32). We assume $\kappa \leq \max \left\{2^{-m / a}, 1 / 2\right\}$. Then, there exists $C>0$, independent of $f$ or $n$, such that

$$
\left\|u-u_{n}\right\|_{H^{1}(\Omega)} \leq C 2^{-m n} \sum_{j=1}^{K}\|f\|_{\mathcal{K}_{a-1}^{m-1}\left(\Omega_{j}\right)} .
$$

Proof. Notice first that $V \subset H^{1}(\Omega)$ continuously, that is, there exists $C>0$ such that $\|v\|_{H^{1}(\Omega)} \leq C\|v\|_{V}$, for all $v \in V$. Also, Céa's Lemma gives that there exists $C_{1}>0$ such that $\left\|u-u_{n}\right\|_{H^{1}(\Omega)} \leq C_{1}\left\|u-\tilde{u}_{I, n}\right\|_{H^{1}(\Omega)}$, where $u_{I, n} \in S_{n}=S\left(\mathcal{T}_{n}, \mathcal{F}\right)$ is the modified interpolant associated to $\mathcal{T}_{n}$ and $\mathcal{F}$, Equation (27). Since $u \in V$, by Theorem 3.1 (quoted from [35]), an application of Theorem 2.21 and of Céa's Lemma give:

$$
\begin{aligned}
\left\|u-u_{n}\right\|_{H^{1}(\Omega)} \leq C\left\|u-u_{I, n}\right\|_{H^{1}(\Omega)} & \leq C\left\|u-u_{I, n}\right\|_{V} \\
& \leq C 2^{-m n}\|u\|_{V} \leq C 2^{-m n} \sum_{j=1}^{K}\|f\|_{\mathcal{K}_{a-1}^{m-1}\left(\Omega_{j}\right)} .
\end{aligned}
$$

The proof is now complete.

A more convenient way of formulating the above theorem may be the following.

Theorem 3.3. Under the notation and assumptions of Theorem 3.2, $u_{n} \in S_{n}$ satisfies

$$
\left\|u-u_{n}\right\|_{H^{1}(\Omega)} \leq C \operatorname{dim}\left(S_{n}\right)^{-m / 2} \sum_{j}\|f\|_{\mathcal{K}_{a-1}^{m-1}\left(\Omega_{j}\right)}
$$

for a constant $C>0$ independent of $f$ and $n$.

Proof. Let again $\mathcal{T}_{n}$ be the triangulation of $\Omega$ after $n$ refinements. Then, the number of triangles is $O\left(4^{n}\right)$ given the refinement procedure of Definition 2.6. Therefore, $\operatorname{dim}\left(S_{n}\right) \simeq 4^{n}$ by Proposition 2.10, so that Theorem 2.21 gives

$$
\left\|u-u_{n}\right\|_{H^{1}(\Omega)} \leq C 2^{-n m} \sum_{j}\|f\|_{\mathcal{K}_{a-1}^{m-1}\left(\Omega_{j}\right)} \leq C \operatorname{dim}\left(S_{n}\right)^{-m / 2} \sum_{j}\|f\|_{\mathcal{K}_{a-1}^{m-1}\left(\Omega_{j}\right)} .
$$

The proof is complete. 
Using that $H^{m-1}(\Omega) \subset \mathcal{K}_{a-1}^{m-1}(\Omega)$ if $a \leq 1$, we obtain the following corollary.

Corollary 3.4. Under the hypotheses of Theorem 3.3,

$$
\left\|u-u_{n}\right\|_{H^{1}(\Omega)} \leq C \operatorname{dim}\left(S_{n}\right)^{-m / 2}\|f\|_{H^{m-1}(\Omega)},
$$

for a constant $C>0$ independent of $f \in H^{m-1}(\Omega)$ and $n$.

Note that we do not claim that $u \in \mathcal{K}_{1}^{1}(\Omega)$ (which is in general not true).

Finally, using a weighted Sobolev space duality argument, we are able to get an estimate of the error in the $L^{2}(\Omega)$ norm.

Theorem 3.5. Under the notation and assumptions of Theorem 2.21, $u_{n} \in S_{n}$ satisfies

$$
\left\|u-u_{n}\right\|_{L^{2}(\Omega)} \leq C \operatorname{dim}\left(S_{n}\right)^{-(m+1) / 2}\|f\|_{H^{m-1}(\Omega)},
$$

for a constant $C>0$ independent of $f$ and $n$.

Proof. Consider the error equation for the bilinear form in (32),

$$
B(\phi, v)=\left(u-u_{n}, v\right), \quad \forall v \in V .
$$

Setting $v=u-u_{n}$ yields,

$$
\left\|u-u_{n}\right\|_{L^{2}(\Omega)}^{2}=\left(u-u_{n}, u-u_{n}\right)=B\left(\phi, u-u_{n}\right) .
$$

Due to the orthogonality of the error in $S_{n}$, we know $B\left(u-u_{n}, v_{n}\right)=0 \quad \forall v_{n} \in S_{n}$. Thus,

$$
\left\|u-u_{n}\right\|_{L^{2}(\Omega)}^{2}=B\left(\phi-\phi_{I, n}, u-u_{n}\right) .
$$

Using the Cauchy-Schwarz inequality gives,

$$
\left\|u-u_{n}\right\|_{L^{2}(\Omega)}^{2} \leq\left\|u-u_{n}\right\|_{H^{1}(\Omega)}\left\|\phi-\phi_{I, n}\right\|_{H^{1}(\Omega)} .
$$

Setting $m=1$ and using the results from Theorem 2.21 (again setting $b=0$ ) with the error equation yields,

$$
\left\|\phi-\phi_{I, n}\right\|_{H^{1}(\Omega)} \leq \operatorname{dim}\left(S_{n}\right)^{-1 / 2}\left\|u-u_{n}\right\|_{L^{2}(\Omega)} .
$$

Therefore, the proof is concluded by using Corollary 3.4 and some simplifications,

$$
\begin{gathered}
\left\|u-u_{n}\right\|_{L^{2}(\Omega)}^{2} \leq\left(C \operatorname{dim}\left(S_{n}\right)^{-m / 2}\|f\|_{H^{m-1}(\Omega)}\right)\left(\operatorname{dim}\left(S_{n}\right)^{-1 / 2}\left\|u-u_{n}\right\|_{L^{2}(\Omega)}\right) \\
\Rightarrow\left\|u-u_{n}\right\|_{L^{2}(\Omega)} \leq C \operatorname{dim}\left(S_{n}\right)^{(-m-1) / 2}\|f\|_{H^{m-1}(\Omega)} .
\end{gathered}
$$

The proof is now complete.

In addition to this, one can improve the regularity estimate of Theorem 3.3 as follows. Choose for each singular point, $Q$, a small neighborhood, $\Omega_{Q}$. Assume that the sets, $\Omega_{Q}$, are disjoint. Then, choose $0<a_{Q}<\eta_{Q}$ such that $\left.u\right|_{\Omega_{Q}} \in$ $\mathcal{K}_{a_{Q}+1}^{m+1}\left(\Omega_{Q}\right)+W_{s}$ if $f \in \mathcal{K}_{a_{Q}-1}^{m-1}\left(\Omega_{Q}\right)$ for all $Q$ and take $0<\kappa_{Q}<2^{-m / a_{Q}}$. For example, if for the Laplacian, $\Delta$, and the same type of boundary conditions (both Dirichlet or both Neumann) on both sides of $Q$, take $a_{Q}<\pi / \alpha_{Q}$, where $\alpha_{Q}$ is the angle at $Q$. On the other hand, if at $Q$ there are different types of boundary conditions, then take $a_{Q}<\pi /\left(2 \alpha_{Q}\right)$. This allows the grading parameter to be controlled better and may lead to better meshes in practice. For instance, this restricts the need of "grading" to a few singular points. 
3.1. Textbook $h^{m}$-estimates. Finally, one can obtain "textbook" $h^{m}$-error estimates as follows. Assume the function $\rho$ is such that $0 \leq \rho \leq 1$ and that $\rho(x)$ is the distance to the singular point $Q$ closest to $x$. Then, there exists an $\epsilon>0$ such that, for any $x$ such that $\rho(x)<\epsilon$, there will be a unique singular point $Q$ closest to $x$. Consider a mesh $\mathcal{T}$ on $\Omega$. For any triangle, $T$, in the given mesh, denote by $d_{T}$ the diameter of the triangle and by $\rho_{T}=\inf _{x \in T} \rho(x)$, which is essentially the distance from $T$ to the closest singular point of $T$. Let $a>0$ be the constant arising in the regularity estimate of Theorem 3.2.

Then, assume that the mesh, $\mathcal{T}$, has the property that there exist constants $C_{0}>0$ and $\alpha>0$ such that

- Any triangle $T$ in the mesh that does not contain a singular point of $\Omega$ has angles $\geq \alpha$ and

$$
d_{T} \leq C_{0} h \rho_{T}^{1-a / m}
$$

- For any triangle $T$ in the mesh that does contain a singular point of $\Omega$, we have

$$
d_{T} \leq C_{0} h^{m / a} .
$$

Denote by $u_{\mathcal{T}} \in S(\mathcal{T}, \mathcal{F})$ the finite-element solution associated to $\mathcal{T}$ and $\mathcal{F}$, then,

$$
\left\|u-u_{\mathcal{T}}\right\|_{H^{1}(\Omega)} \leq C_{1} h^{m} \sum_{j}\|f\|_{\mathcal{K}_{a-1}^{m-1}\left(\Omega_{j}\right)},
$$

with a constant $C_{1}$ that depends only on $C_{0}$ and $\alpha$. Provided that one constructs a mesh with "few" triangles, then the above estimate can be used to recover Theorem 3.3 by using estimates analogous to Equations (29) and (23).

\section{Conclusion}

We have shown that by using a sequence of graded meshes, optimal approximation results can be regained for mixed boundary value/transmission problems of type (1), whose solutions contain singularities. General conformally invariant families of finite elements with high order can also be used in the context of these graded meshes and weighted spaces. Thus, for problems that require higher regularity or smoothness of the finite-element spaces, such as for problems of high order or those requiring higher order $p$-refinement, optimal results can still be obtained. Future work involves extending these results to the least-squares finite-element method applied to problems with corner singularities [19, 24, 33, 32]. In these applications, the addition of the graded meshes can be used to show that the least-squares functional does in fact predict the optimal rate of convergence of the finite-element method. Another natural problem is to study the Multigrid method for the resulting spaces. See [15] for results in this direction.

\section{REFERENCES}

[1] T. Apel and S. Nicaise. The finite element method with anisotropic mesh grading for elliptic problems in domains with corners and edges. Math. Methods Appl. Sci., 21(6):519-549, 1998.

[2] T. Apel, A.-M. Sändig, and J. Whiteman. Graded mesh refinement and error estimates for finite element solutions of elliptic boundary value problems in non-smooth domains. Math. Methods Appl. Sci., 19(1):63-85, 1996.

[3] T. Apel and J. Schöberl. Multigrid methods for anisotropic edge refinement. SIAM J. Numer. Anal., 40(5):1993-2006 (electronic), 2002.

[4] D. Arnold and R. Falk. Well-posedness of the fundamental boundary value problems for constrained anisotropic elastic materials. Arch. Rational Mech. Anal., 98(2):143-165, 1987. 
[5] D. Arnold, R. Falk, and R. Winther. Finite element exterior calculus, homological techniques, and applications. Acta Numer., 15:1-155, 2006.

[6] D. Arnold, R. Falk, and R. Winther. Finite element exterior calculus: from Hodge theory to numerical stability. Bull. Amer. Math. Soc. (N.S.), 47(2):281-354, 2010.

[7] I. Babuška. Finite element method for domains with corners. Computing (Arch. Elektron. Rechnen), 6:264-273, 1970.

[8] I. Babuška and A. K. Aziz. Survey lectures on the mathematical foundations of the finite element method. In The mathematical foundations of the finite element method with applications to partial differential equations (Proc. Sympos., Univ. Maryland, Baltimore, Md., 1972), pages 1-359. Academic Press, New York, 1972. With the collaboration of G. Fix and R. B. Kellogg.

[9] I. Babuška, R. B. Kellogg, and J. Pitkäranta. Direct and inverse error estimates for finite elements with mesh refinements. Numer. Math., 33(4):447-471, 1979.

[10] C. Bacuta, J. H. Bramble, and J. Xu. Regularity estimates for elliptic boundary value problems with smooth data on polygonal domains. J. Numer. Math., 11(2):75-94, 2003.

[11] C. Bacuta, J.H. Bramble, and J Xu. Regularity estimates for elliptic boundary value problems in Besov spaces. Math. Comp., 72:1577-1595, 2003.

[12] C. Bacuta, V. Nistor, and L. Zikatanov. Improving the rate of convergence of 'high order finite elements' on polygons and domains with cusps. Numerische Matemathik, 100:165-184, 2005.

[13] M. Berndt, T. Manteuffel, S. McCormick, and G. Starke. Analysis of first-order system least squares (FOSLS) for elliptic problems with discontinuous coefficients. I. SIAM J. Numer. Anal., 43(1):386-408 (electronic), 2005.

[14] J. Bramble and J. King. A finite element method for interface problems in domains with smooth boundaries and interfaces. Advances in Computational Mathematics, 6:109-138, 1996. 10.1007/BF02127700.

[15] J. Brannick, H. Li, and L. Zikatanov. Uniform convergence of the multigrid $V$-cycle on graded meshes for corner singularities. Numer. Linear Algebra Appl., 15(2-3):291-306, 2008.

[16] S. Brenner and M. Neilan. A $C^{0}$ interior penalty method for a fourth order elliptic singular perturbation problem. SIAM J. Numer. Anal., 49(2):869-892, 2011.

[17] S. Brenner and R. Scott. The mathematical theory of finite element methods, volume 15 of Texts in Applied Mathematics. Springer-Verlag, New York, second edition, 2002.

[18] Susanne C. Brenner and Li-Yeng Sung. $C^{0}$ interior penalty methods for fourth order elliptic boundary value problems on polygonal domains. J. Sci. Comput., 22/23:83-118, 2005.

[19] Z. Cai, R. Lazarov, T. A. Manteuffel, and S. F. McCormick. First-order system least squares for second-order partial differential equations. I. SIAM J. Numer. Anal., 31(6):1785-1799, 1994.

[20] L. Chen and C. Zhang. A coarsening algorithm on adaptive grids by newest vertex bisection and its applications. J. Comput. Math., 28(6):767-789, 2010.

[21] P. Ciarlet. The finite element method for elliptic problems. North-Holland Publishing Co., Amsterdam, 1978. Studies in Mathematics and its Applications, Vol. 4.

[22] P. Ciarlet. Basic error estimates for elliptic problems. In Handbook of numerical analysis, Vol. II, Handb. Numer. Anal., II, pages 17-352. North-Holland, Amsterdam, 1991.

[23] M. Costabel, M. Dauge, and C. Schwab. Exponential convergence of $h p$-FEM for Maxwell equations with weighted regularization in polygonal domains. Math. Models Methods Appl. Sci., 15(4):575-622, 2005.

[24] C. L. Cox and G. J. Fix. On the accuracy of least squares methods in the presence of corner singularities. Comput. Math. Appl., 10(6):463-475 (1985), 1984.

[25] M. Dauge. Elliptic boundary value problems on corner domains, volume 1341 of Lecture Notes in Mathematics. Springer-Verlag, Berlin, 1988. Smoothness and asymptotics of solutions.

[26] P. Grisvard. Elliptic problems in nonsmooth domains, volume 24 of Monographs and Studies in Mathematics. Pitman (Advanced Publishing Program), Boston, MA, 1985.

[27] P. Grisvard. Singularities in boundary value problems, volume 22 of Research in Applied Mathematics. Masson, Paris, 1992.

[28] B. Guo and I. Babuška. Regularity of the solutions for elliptic problems on nonsmooth domains in $\mathbf{R}^{3}$. I. Countably normed spaces on polyhedral domains. Proc. Roy. Soc. Edinburgh Sect. A, 127(1):77-126, 1997. 
[29] J. Guzman, D. Leykekhman, and M. Neilan. A family of non-conforming elements and the analysis of nitsche's method for a singularly perturbed fourth order problem. Calcolo, 49(2):95-125, 2012.

[30] V. A. Kondrat'ev. Boundary value problems for elliptic equations in domains with conical or angular points. Transl. Moscow Math. Soc., 16:227-313, 1967.

[31] V. Kozlov, V. Maz'ya, and J. Rossmann. Spectral problems associated with corner singularities of solutions to elliptic equations, volume 85 of Mathematical Surveys and Monographs. American Mathematical Society, Providence, RI, 2001.

[32] E. Lee, T. A. Manteuffel, and C. R. Westphal. Weighted-norm first-order system least squares (FOSLS) for problems with corner singularities. SIAM J. Numer. Anal., 44(5):1974-1996 (electronic), 2006.

[33] E. Lee, T. A. Manteuffel, and C. R. Westphal. Weighted-norm first-order system least-squares (FOSLS) for div/curl systems with three dimensional edge singularities. SIAM J. Numer. Anal., 46(3):1619-1639, 2008.

[34] H. Li. Finite element analysis for the axisymmetric Laplace operator on polygonal domains. J. Comput. Appl. Math., 235(17):5155-5176, 2011.

[35] H. Li, A. Mazzucato, and V. Nistor. Analysis of the finite element method for transmission/mixed boundary value problems on general polygonal domains. Electron. Trans. Numer. Anal., 37:41-69, 2010.

[36] G. Raugel. Résolution numérique par une méthode d'éléments finis du problème de Dirichlet pour le laplacien dans un polygone. C. R. Acad. Sci. Paris Sér. A-B, 286(18):A791-A794, 1978.

[37] C. Schwab. P-And Hp- Finite Element Methods: Theory and Applications in Solid and Fluid Mechanics. Clarendon Press, 1998.

[38] G. Strang and G. Fix. An analysis of the finite element method. Wellesley-Cambridge Press, Wellesley, MA, second edition, 2008.

James Adler, Math. Dept., Tufts University, Medford, MA 02155

E-mail address: james.adler@tufts.edu

Victor Nistor, Math. Dept., Penn State University, University Park, PA 16802

E-mail address: nistor@math.psu.edu 